\title{
Searching for relic neutralinos using neutrino telescopes
}

\author{
V. Berezinsky ${ }^{\mathrm{a}, 1}$, A. Bottino ${ }^{\mathrm{b}, \mathrm{c}, 2}$, J. Ellis ${ }^{\mathrm{d}, 3}$, N. Fornengo ${ }^{\mathrm{c}, \mathrm{e}, 4}$, G. Mignola $^{\mathrm{c}, \mathrm{d}, \mathrm{S}}$, \\ S. Scopel ${ }_{\mathrm{f}, \mathrm{g}, 6}$ \\ a INFN, Laboratori Nazionali del Gran Sasso, 67010 Assergi (AQ), Italy \\ ${ }^{\mathrm{b}}$ Dipartimento di Fisica Teorica, Università di Torino, Via P. Giuria 1, 10125 Torino, Italy \\ c INFN, Sezione di Torino, Via P. Giuria 1, 10125 Torino, Italy \\ 'Theoretical Physics Division, CERN, CH-1211 Geneva 23, Switzerland \\ 'Department of Physics and Astronomy, The Johns Hopkins University, Baltimore, MD 21218, USA \\ f Dipartimento di Fisica, Università di Genova, Via Dodecaneso 33, 16146 Genova, Italy \\ INFN, Sezione di Genova, Via Dodecaneso 33, 16146 Genova, Italy
}

Received 20 March 1996

\begin{abstract}
Neutrino telescopes of large area offer the possibility of searching for indirect signals of relic neutralinos in the galactic halo, due to annihilations in the Sun or the Earth. Here we investigate the sensitivity, using a supergravity scheme where the soft scalar mass terms are not constrained by universality conditions at the grand unification scale. We first discuss in which regions of the supersymmetric parameter space the neutralino may be considered as a good candidate for cold dark matter. The discovery potential of the search using neutrino telescopes is then compared to that of the direct search for relic neutralinos.
\end{abstract}

\section{Introduction}

In large regions of the supersymmetric parameter space the neutralino turns out to be the Lightest Supersymmetric Particle (LSP) and, as such, it is stable, provided R-parity is conserved. Under these hypotheses the neutralino would have decoupled from the initial plasma in the early stages of the Universe, and would now be present as a relic particle [1].

\footnotetext{
${ }^{1}$ E-mail: berezinsky@lngs.infn.it.

${ }^{2}$ E-mail: bottino@to.infn.it.

${ }^{3}$ E-mail: johne@cemvm.cern.ch.

${ }^{4}$ E-mail: fornengo@jhup.pha.jhu.edu.

${ }^{5}$ E-mail: mignola@to.infn.it.

${ }^{6}$ E-mail: scopel@ge.infn.it.
}

The LSP is a candidate for the Cold Dark Matter (CDM) that is believed to have played a key role in the formation of structures in the Universe, such as galaxies and clusters. Although there are other candidates for CDM, such as the axion or the axino, and scenarios for structure formation that do not involve a large density of CDM, the possibility that the neutralino provides most of the CDM remains in our opinion the most attractive option. The 'standard model' of structure formation used to be one with an initially flat Harrison-Zeldovich spectrum of inflationary fluctuations and (essentially) the critical density of CDM: $\Omega_{\mathrm{CDM}} \equiv \rho_{\mathrm{CDM}} / \rho_{\text {crit }} \simeq 1$. However, the advent of COBE and other data have suggested that this model needs to be modified, and there are three main contenders on the market [2]. 
One is a 'mixed' dark matter (or Cold Hot Dark Matter) model (CHDM), in which $\Omega_{\mathrm{CDM}} \simeq 0.7$, there is a hot dark matter component with $\Omega_{\mathrm{HDM}} \simeq 0.2$, and baryons contribute $\Omega_{\mathrm{B}} \leq 0.1$, as reviewed in Section 2. Another is a model ( $A C D M)$ with a significant cosmological constant $\Omega_{\Lambda} \simeq 0.7$ and $\Omega_{\mathrm{CDM}} \simeq 0.3$. Finally, we mention a cold dark matter with a tilted spectrum of initial fluctuations (TCDM), in which $\Omega_{\mathrm{CDM}} \simeq 1$ is still possible, and an open model with $\Omega_{\mathrm{CDM}} \simeq 0.3$. Calculations of the relic LSP abundance actually lead to values for the product $\Omega_{\mathrm{LSP}} h^{2}$, where $h$ is the present Hubble expansion rate $H_{0}$ in units of $100 \mathrm{~km} \mathrm{~s}^{-1} \mathrm{Mpc}^{-1}$. There is still some observational uncertainty in this quantity, which may lie in the range $0.5 \leq h \leq 0.9$. Within this range, the age of the Universe favours smaller values of $h$ in the CHDM and TCDM scenarios, whereas larger values are possible in the $\Lambda$ CDM scenario. Combining the estimates of $\Omega_{\mathrm{CDM}}$ and $h$ in each of the three scenarios, we find the preferred range

$\Omega_{\mathrm{CDM}} h^{2}=0.2 \pm 0.1$

However, we repeat that not all the CDM need be constituted of LSPs, so $\Omega_{\text {LSP }}$ could in principle lie below the range (1).

Even if relic neutralinos do not provide a significant fraction of dark matter, experimental evidence for them would add a relevant new piece of information on the early stages of the Universe. Various strategies for detection of relic neutralinos are currently being pursued ${ }^{7}$. The most straightforward technique (direct detection) consists in measuring the effect that an impinging neutralino may produce in an appropriate detector by its elastic scattering off a target nucleus [6] ${ }^{8}$. Among the indirect ways of detecting relic neutralinos, one of the most promising ones is the observation, using neutrino telescopes of large area, of the up-going muons which would be generated by neutrinos produced by pair annihilation

\footnotetext{
${ }^{7}$ For reviews on detection methods for dark matter candidates, see Ref. [3]. An updated review of the phenomenology of neutralino dark matter is presented in Ref. [4].

${ }^{8}$ For an updated list of references on this topic, which would be too long to report here, we refer to the references quoted in Jungman, Kamionkowski and Griest (see Refs. [4-6]).
}

of neutralinos captured and accumulated inside celestial bodies such as the Earth and the Sun $[7,8]^{9}$.

At present, theory is unfortunately unable to offer firm predictions for the event rates for detection of relic neutralinos, since supersymmetric theories are still awaiting experimental verification. Only some hints for possible supersymmetric effects are available from accelerator data: supersymmetric theories would favor the unification feature of the gauge running constants [10] and the apparently relatively light Higgs boson mass [11]. Apart from these properties, the physical (correct) supersymmetric scheme is not known yet, and thus one has to consider a number of various possible scenarios. The detection rates for neutralinos depend very sensitively on the different supersymmetric schemes employed in the analysis.

The least constrained theoretical model is represented by the Minimal Supersymmetric Extension of the Standard Model (MSSM), which incorporates the same gauge group as the standard model and the minimal supersymmetric extension of its particle content [12]. The Higgs sector contains two doublets $H_{1}, H_{2}$ which give masses to down- and up-type quarks, respectively. This scheme provides a very useful framework for analyzing the phenomenology at the $M_{Z}$ scale with a minimal number of model-dependent restrictions. The main inconvenience of this approach is that one typically has to deal with a large number of free parameters.

Much more ambitious are theoretical schemes where features at the $M_{Z}$ scale are derived from properties at the Grand Unification (GU) scale ( $M_{\mathrm{GUT}}$ ), the link being provided by the Renormalization Group Equations (RGE's). One of the most attractive supersymmetric models is the one in which Electro-Weak Symmetry Breaking (EWSB) is induced radiatively [13]. This model is in no way the only possible model, but has the very nice feature of connecting the EWSB to soft supersymmetry breaking. Furthermore, the requirement of radiative EWSB is effective in reducing the number of the free parameters.

\footnotetext{
${ }^{9}$ For a review of the new experimental projects of neutrino telescopes, see Ref. [9].
} 
In order to constrain the scheme further, one often makes a number of rather restrictive hypotheses. Typically one assumes that not only the gauge couplings but also the Yukawa couplings of $b$ and $\tau$ and the soft-breaking mass parameters (gaugino masses, scalar masses and trilinear couplings) unify at a GUT scale $M_{\mathrm{GUT}}=O\left(10^{16} \mathrm{GeV}\right)$. These assumptions entail very strong consequences for neutralino phenomenology, and in particular for the properties of neutralino dark matter. However, it has been shown that many aspects of neutralino phenomenology may change quite significantly, if one relaxes the universality requirements $[14,15]$. This possibility has been explored in Ref. [6] in an analysis of the neutralino relic abundance and of the event rates for direct detection in a wide range of the supersymmetric parameter space.

In the present paper we extend the analysis of Ref. [6] to the evaluation of the event rates for indirect relic neutralino searches using neutrino telescopes. Our results are compared with new, more stringent experimental bounds, obtained using the Baksan detector [16]. Furthermore, we also present a comparison between the discovery potential of searches using neutrino telescopes and the direct method.

The plan of the paper is as follows. In Section 2 we expand the above discussion of the CDM density, and derive the estimate (1) for $\Omega_{\mathrm{CDM}} h^{2}$, by considering various cosmological models. The main features of the supersymmetric scheme employed in this paper are briefly described in Section 3, and in Section 4 we present our results on the neutralino relic abundance. Section 5 is devoted to the derivation of the flux of up-going muons due to neutralino-neutralino annihilation in the Earth and in the Sun. In Section 5 we also discuss some relevant features of the experimental layout required for the detection of the signals under study. Finally, results and conclusions are given in Section 6.

\section{Density of CDM in cosmological models}

Here we consider the neutralino as a CDM particle in the framework of several cosmological models. Such models are primarily characterized by two dimensionless parameters $h=H_{0} /\left(100 \mathrm{~km} \mathrm{~s}^{-1}\right.$
$\mathrm{Mpc}^{-1}$ ) and $\Omega=\rho / \rho_{\text {crit }}$, where $\rho$ is the relic cosmological density and $\rho_{\text {crit }} \simeq 1.88 \cdot 10^{-29} h^{2} \mathrm{~g} / \mathrm{cm}^{3}$ is the critical density.

Different measurements of the Hubble constant imply $0.4 \leq h \leq 1$ [17], but the recent measurements of extragalactic Cepheids in the Virgo [18] and Coma [19] clusters have narrowed this interval to $0.5 \leq h \leq$ 0.9 . However, this range should be taken with some caution, because of the uncertainties involved in these difficult measurements. In particular, the value $h=0.5$ has to be considered as only marginally allowed. Inspired mostly by theoretical motivations (flatness problem and the beauty of the inflationary scenario), $\Omega=1$ is usually assumed. This value is consistent with the IRAS [20] data and the POTENT [21] analysis, and no observational data contradict this value significantly.

Dark matter can be subdivided into baryonic DM, hot DM (HDM) and CDM. The density of baryonic matter found from nucleosynthesis is taken [22] as $\Omega_{\mathrm{B}} h^{2}=0.025 \pm 0.005$. The other DM components are defined as hot or cold components depending on their velocities at the moment when galaxies cross the horizon scale. If particles are relativistic they are called HDM particles, if not, CDM particles. The natural candidate for HDM is the heaviest neutrino, most probably the $\tau$ neutrino. Structure formation in the Universe puts strong restrictions to the properties of DM in the Universe. A Universe with only HDM and baryonic DM gives a wrong prediction for the spectrum of fluctuations as compared with the measurements of COBE [23], IRAS [20] and the CfA [24] survey. CDM and baryonic matter alone may explain the spectrum of fluctuations if the total density $\Omega \simeq 0.3$. There is one more possible form of energy density in the Universe, namely the vacuum energy described by the cosmological constant $\Lambda$. The corresponding energy density is given by $\Omega_{A}=$ $\Lambda /\left(3 H_{0}^{2}\right)$. Quasar lensing and the COBE results restrict the vacuum energy density to $\Omega_{A} \leq 0.7$ [25].

There are several cosmological models based on the four types of DM described above (baryonic DM, HDM, CDM and vacuum energy). These models predict different spectra of fluctuations to be compared with the data of COBE, IRAS, the CfA survey, etc. They also produce different cluster-cluster correlations, number densities of clusters, velocity dispersions and other properties. The simplest and most 
attractive model for a correct description of all these phenomena is the mixed model (CHDM). This model is characterized by the following parameters:

$\Omega_{\Lambda}=0, \quad \Omega_{0}=\Omega_{\mathrm{B}}+\Omega_{\mathrm{CDM}}+\Omega_{\mathrm{HDM}}=1$,

$H_{0} \simeq 50 \mathrm{~km} \mathrm{~s}^{-1} \mathrm{Mpc}^{-1}(h \simeq 0.5)$,

$\Omega_{\mathrm{CDM}}: \Omega_{\mathrm{HDM}}: \Omega_{\mathrm{B}} \simeq 0.7: 0.2: 0.1$,

where $\Omega_{\mathrm{HDM}} \simeq 0.2$ is obtained in Ref. [26] from data on damped Lyman $\alpha$ clouds. In this CHDM model the central value for the CDM density is given by

$\Omega_{\mathrm{CDM}} h^{2}=0.18$

with uncertainties which may be estimated as $\leq$ \pm 0.1 . As already mentioned, the best candidate for the HDM particle is the $\tau$ neutrino, and in the CHDM model with $\Omega_{\nu}=0.2$ its mass should be $m_{\nu_{\tau}} \simeq 4.7 \mathrm{eV}$. A very good fit to the cosmological data is given by a CHDM model where the HDM is constituted of two neutrinos $\left(\mathrm{C} \nu^{2} \mathrm{DM}[27,28]\right)$. In our view, in either case, the most plausible candidate for the CDM particle is probably the neutralino $(\chi)$.

In the light of recent measurements of the Hubble constant, the CHDM model faces a possible age problem. A lower limit on the age of the Universe $t_{0} \geq 13 \mathrm{Gyr}$ from globular clusters imposes an upper limit on the Hubble constant in the CHDM model: $H_{0} \lesssim 50 \mathrm{~km} \mathrm{~s}^{-1} \mathrm{Mpc}^{-1}$. This value is in slight contradiction with the recent observations of extragalactic Cepheids, which can be summarized as $H_{0}$ $\geq 60 \mathrm{~km} \mathrm{~s}^{-1} \mathrm{Mpc}^{-1}$. However, it is too early to consider this as a serious conflict [29], if we take into account the many uncertainties and physical possibilities (e.g., the Universe can be locally overdense see the discussion in Ref. [28]).

The age problem, if taken seriously, can be solved with the help of another successful cosmological model, $\Lambda$ CDM. This model assumes that $\Omega=1$ is provided by the vacuum energy (cosmological constant $\Lambda$ ) and CDM. In this case we have $\Omega_{\Lambda} \simeq 0.7$, $\Omega_{\mathrm{CDM}} \approx 0.3$ and $h \leq 0.7$. Thus this model predicts $\Omega_{\mathrm{CDM}} h^{2} \simeq 0.15$ with an uncertainty of order 0.1 .

Finally, we mention two other CDM models. The first one is the tilted CDM model (TCDM), where the initial fluctuation spectrum is steeper than the Harrison-Zeldovich spectrum, and the second one is the CDM model with $\Omega=\Omega_{\mathrm{CDM}}=0.3$ (CDM). Both models give a good fit to the observed spectrum of
Table 1

$\Omega_{\mathrm{CDM}} h^{2}$ for five cosmological models

\begin{tabular}{llll}
\hline & $\Omega_{\mathrm{CDM}}$ & $h$ & $\Omega_{\mathrm{CDM}} h^{2}$ \\
\hline CHDM & 0.7 & 0.5 & 0.18 \\
$\Lambda \mathrm{CDM}$ & 0.3 & $0.7-0.8$ & $0.15-0.19$ \\
$C \nu^{2} \mathrm{DM}$ & 0.7 & 0.5 & 0.18 \\
TCDM & 1.0 & 0.5 & 0.25 \\
CDM & 0.3 & $0.7-0.8$ & $0.15-0.19$ \\
\hline
\end{tabular}

fluctuations as well as good agreement with the cluster data, though they may conflict with conventional inflationary prejudices.

In Table 1 we summarize the estimates of $\Omega_{\mathrm{CDM}}$ for all these models and the ensuing values for $\Omega_{\mathrm{CDM}} h^{2}$. Taking into account the uncertainties, we conclude as mentioned in Section 1 that, for all the cosmological models considered here

$\Omega_{\mathrm{CDM}} h^{2}=0.2 \pm 0.1$

In the following we will emphasize regions of the supersymmetric parameter space which yield a neutralino relic abundance $\Omega_{\chi} h^{2}$ within the range of Eq. (4), but consider also regions with lower $\Omega_{\chi} h^{2}$, since there can be additional forms of CDM, such as axions.

\section{Theoretical framework}

We turn now to a short presentation of the theoretical model employed here to describe the neutralino. We adopt a supersymmetric model whose essential elements are provided by Yang-Mills Lagrangian, the superpotential, which contains all the Yukawa interactions between the standard and supersymmetric fields, and by a soft-breaking Lagrangian with the usual trilinear couplings (with parameters $A_{i}$ ), the Higgs-mixing term $\mu$, and the mass terms ( $M_{i}$ for the gaugino masses and $m_{i}$ for the scalar masses). While unification conditions at $M_{\mathrm{GUT}}$ are imposed on gaugino masses: $M_{i}\left(M_{\mathrm{GUT}}\right) \equiv m_{1 / 2}$, and for the trilinear couplings ( $A_{0}$ being their common value at $M_{\mathrm{GUT}}$ ), soft scalar masses are allowed to deviate from strict universality. We recall that gaugino masses are renormalized in the same way as gauge couplings, so the consistency of the measured values of the latter with supersymmetric unification 
motivates similar unification of the gaugino masses, as assumed here (for a more detailed discussion, see our previous paper [6]). More specifically, we consider here a departure from universality in the scalar masses at $M_{\mathrm{GUT}}$ that splits the soft-supersymmetrybreaking mass parameters of the two Higgs doublets $M_{\mathrm{H}_{1}}, M_{\mathrm{H}_{2}}$ in the following way

$M_{\mathrm{H}_{i}}^{2}\left(M_{\mathrm{GUT}}\right)=m_{0}^{2}\left(1+\delta_{i}\right)$.

The parameters $\delta_{i}$ are varied in the range $(-1$, +1 ), but are taken to be independent of the other supersymmetric parameters.

Our supersymmetric parameter space is then constrained by a number of conditions: (a) all experimental bounds on Higgs, neutralino, chargino and sfermion masses are satisfied (taking into account also the new data from LEP1.5 [30], (b) the neutralino is the lightest supersymmetric particle (LSP), (c) constraints on the $b \rightarrow s \gamma$ process and on the mass of the bottom quark $m_{b}$ assuming $b-\tau$ Yukawa unification are satisfied, (d) EWSB is realized radiatively, (e) radiative EWSB occurs without excessive fine-tuning, (f) the neutralino relic abundance does not exceed the cosmological bound. In particular, the requirements of radiative EWSB and of the universality conditions on the gaugino masses and on the trilinear couplings allow a reduction of the independent parameters to the following set (apart from the $\delta_{i}$ 's): $m_{1 / 2}, m_{0}, A_{0}, \tan \beta\left(\tan \beta\right.$ is the ratio $v_{2} / v_{1}$ of the vacuum expectation values of the two Higgs doublets). It has to be emphasized that, because of the assumption of radiative EWSB, the parameter $\mu$ of the Higgs-mixing term in the superpotential is not a further independent parameter, but is a function of the previous set of parameters.

As we emphasized in our previous paper [6], attention must be paid to the condition that radiative EWSB is satisfied without excessive tuning [31,32]. In Ref. [6] we found that requiring accidental cancellations among various competing terms not to exceed the $1 \%$ level sets the bound $m_{x} \lesssim 200 \mathrm{GeV}$. Further details of the theoretical scheme adopted here can be found in Ref. [6].

A departure from $m_{0}$ universality of the type given in Eq. (5) may modify the neutralino phenomenology in a significant way. Two key parameters whose values may change sizably, as functions of the $\delta_{i}$ 's, are $\mu$ and the mass $m_{A}$ of the CP-odd Higgs boson $A$. In turn, variations in $\mu$ and $M_{A}$ may induce significant modifications in the neutralino properties. It is convenient to express these two parameters in the following way [6]

$$
\begin{aligned}
\mu^{2}= & J_{1} m_{1 / 2}^{2}+J_{2} m_{0}^{2}+J_{3} A_{0}^{2} m_{0}^{2}+J_{4} A_{0} m_{0} m_{1 / 2} \\
& -\frac{1}{2} M_{Z}^{2}
\end{aligned}
$$

and

$$
\begin{aligned}
M_{A}^{2}= & K_{1} m_{1 / 2}^{2}+K_{2} m_{0}^{2}+K_{3} A_{0}^{2} m_{0}^{2}+K_{4} A_{0} m_{0} m_{1 / 2} \\
& -M_{Z}^{2},
\end{aligned}
$$

where the coefficients $J_{i}$ and $K_{i}$, which are functions of $\tan \beta$ and of the $\delta_{i}$ (except for $J_{1}$ and $K_{1}$ which depend on $\tan \beta$ only), are obtained from the RGE's. The coefficients $J_{1}, J_{2}$ and $K_{1}, K_{2}$ are given in Fig. 1 for the case of $m_{0}$ universality. From now on we will set $A_{0}=0$, thus the other coefficients are irrelevant for our discussion. Except for very small values of $\tan \beta(\tan \beta \leqq 4)$, one has $J_{2} \ll J_{1}$, which in turn implies a strong $m_{1 / 2}-\mu$ correlation and a gaugino-like neutralino. However, even moderate departures from $m_{0}$ universality may modify this picture $[6,14]$. For instance, at large $\tan \beta$, non vanishing values of the $\delta_{i}$ 's may yield a sizeable value of $\left|J_{2}\right|$, which in turn entails either a more pronounced gaugino-like neutralino, when $J_{2}$ $>0$, or a mixed higgsino-gaugino composition for the neutralino, when $J_{2}<0$.

Also the coefficient $K_{2}$ plays a key role in establishing important phenomenological properties. In the universal case (see Fig. 1), $K_{2}$ is positive and sizeable, except at very large $\tan \beta$ (i.e., $\tan \beta \approx 50$ ). Thus, $M_{A}$ turns out to be large except at very large values of $\tan \beta$, where it may approach the present experimental bound $M_{A} \gtrsim 55 \mathrm{GeV}$. Again, deviations from $m_{0}$ universality may modify $K_{2}$ substantially and thus may change the value of $M_{A}$, too. We will see in the following how these properties affect the values of some important quantities, such as the relic abundance.

For further details about the procedure we adopted to solve the RGE's and to implement the constraints due to $b \rightarrow s \gamma$ and to $m_{b}$, we refer to our previous paper [6]. Here we only recall that we used 1-loop beta functions including the whole supersymmetric particle spectrum from the GUT scale down to $M_{Z}$, 

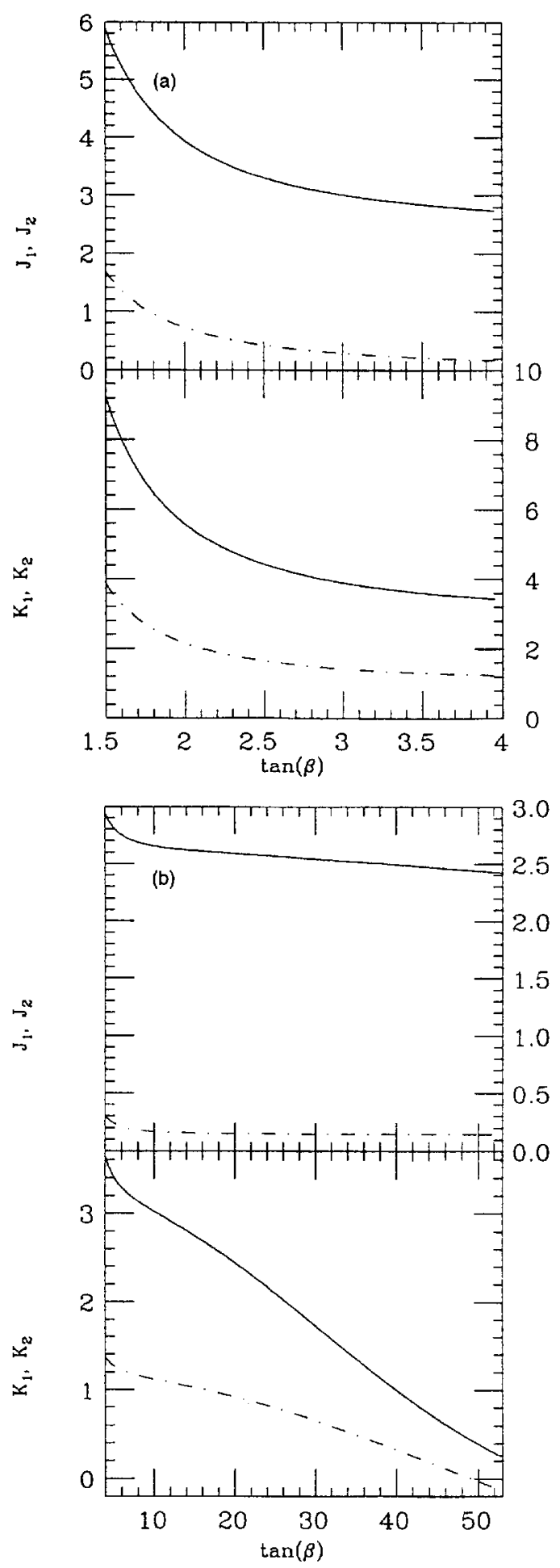

neglecting the possible effects of intermediate thresholds. Two-loop and threshold effects, which are known to be of key importance for specific refined calculations, such as the unification of the gauge couplings, were not included, since we are interested in overall neutralino properties studied over a wide range of variation for the high-scale parameters. We have allowed generous ranges for $b \rightarrow s \gamma$ and $m_{b}$, to accommodate uncertainties in QCD corrections and the correct Yukawa unification condition, respectively. Furthermore, some physical solutions are unstable with respect to allowable variations in the strong gauge coupling constant $\alpha_{s}$. Although the best way of proceeding would be to allow $\alpha_{s}$ to vary over its whole physical range, here, for simplicity, we have preferred to show our results only for some representative values of $\alpha_{s}$. In one case in Section 4 we show comparatively our results for two different values of the strong coupling constant.

Our results for $\Omega_{\chi} h^{2}$ and for the detection rates have been obtained by varying the parameters $m_{0}$ and $m_{1 / 2}$ on an equally-spaced linear grid over the ranges $10 \mathrm{GeV} \leq m_{0} \leq 2 \mathrm{TeV}, 45 \mathrm{GeV} \leq m_{1 / 2} \leq 500$ $\mathrm{GeV}$ at fixed $\tan \beta$, with $A_{0}=0$. Furthermore, we remark that all evaluations presented here are for positive values of $\mu$, since negative values of $\mu$ are disfavoured by the constraints due to $m_{b}$ and the $b \rightarrow s \gamma$ process.

As far as the values of $\tan \beta$ are concerned, we note that recent global fits of the electroweak data within the MSSM [33] focused interest on two narrow intervals for $\tan \beta$ : (i) very small values, $\tan \beta$ $\simeq 1-2$ (i.e., close to the quasi-infrared fixed point for a given value of $m_{t}$ ), or (ii) very large values of $\tan \beta$ (i.e., of order $m_{t} / m_{b}$ ). In the present paper, as representative values for case (i) and case (ii), we take the values $\tan \beta=1.5$ and $\tan \beta=53$, respectively.
Fig. 1. Coefficients $J_{1}, J_{2}$ and $K_{1}, K_{2}$ of the polynomial expressions $(6,7)$ as functions of $\tan \beta$. The range $1.5 \leq \tan \beta \leq 4$ is shown in section (a) and the range $4 \leq \tan \beta \leq 53$ in section (b). In the upper part $J_{1}$ is denoted by a solid line, $J_{2}$ by a dot-dashed line. In the lower part, $K_{1}$ is denoted by a solid line, $K_{2}$ by a dot-dashed line. 
We conclude this section by summarizing the basic features of our model [6]: (i) the universality condition for the scalar masses is relaxed, (ii) rather relaxed restrictions from $b \rightarrow s \gamma$ and the mass of the $b$ quark are allowed, (iii) the fine-tuning condition limits the neutralino mass to $m_{x} \leq 200 \mathrm{GeV}$.

\section{Neutralino relic abundance}

For the evaluation of $\Omega_{\chi} h^{2}$ we have followed the standard method [34-37]. We recall that $\Omega_{\chi} h^{2}$ is essentially given by $\Omega_{\chi} h^{2} \propto\left\langle\sigma_{\mathrm{ann}} v\right\rangle_{\text {int }}^{-1}$, where $\left\langle\sigma_{\mathrm{ann}} v\right\rangle_{\mathrm{int}}$ is the thermally-averaged annihilation cross section, integrated from the freeze-out temperature to the present temperature. Then the key quantity to be evaluated is the annihilation cross-section.

In the evaluation of $\sigma_{\mathrm{ann}}$ we have considered the following set of final states: (1) fermion-antifermion pairs, (2) pairs of Higgs bosons, (3) one Higgs boson and one gauge boson, (4) pairs of gauge bosons. For the final state (1), the following diagrams have been considered: Higgs- and Z-exchange diagrams in the $s$ channel and $\tilde{f}$ exchange in the $t$ channel. For the final states ( 2 to 4 ) we have included Higgs-exchange and $Z$-exchange diagrams in the $s$ channel, and either neutralino (the full set of the four mass eigenstates) or chargino exchange in the $t$ channel, depending on the electric charges of the final particles [37].

The relative importances of the various exchange diagrams depend on the supersymmetric parameters through the couplings and masses of the exchanged particles. Typically, one expects a small value of $\sigma_{\text {ann }}$ at $\operatorname{small} \tan \beta$, where $\sigma_{\text {ann }}$ is dominated by sfermion and $Z$ exchanges. In fact, in supergravity models at small $\tan \beta$, Higgs-exchange contributions are reduced not only by small couplings, but also by large values of the mass $M_{A}$. These features are displayed in Fig. 2, which is for $\tan \beta=1.5$ and $\delta_{1}=\delta_{2}=0$. As expected, $\Omega_{\chi} h^{2}$ is rather large, and many configurations are excluded by the cosmologi- (a)

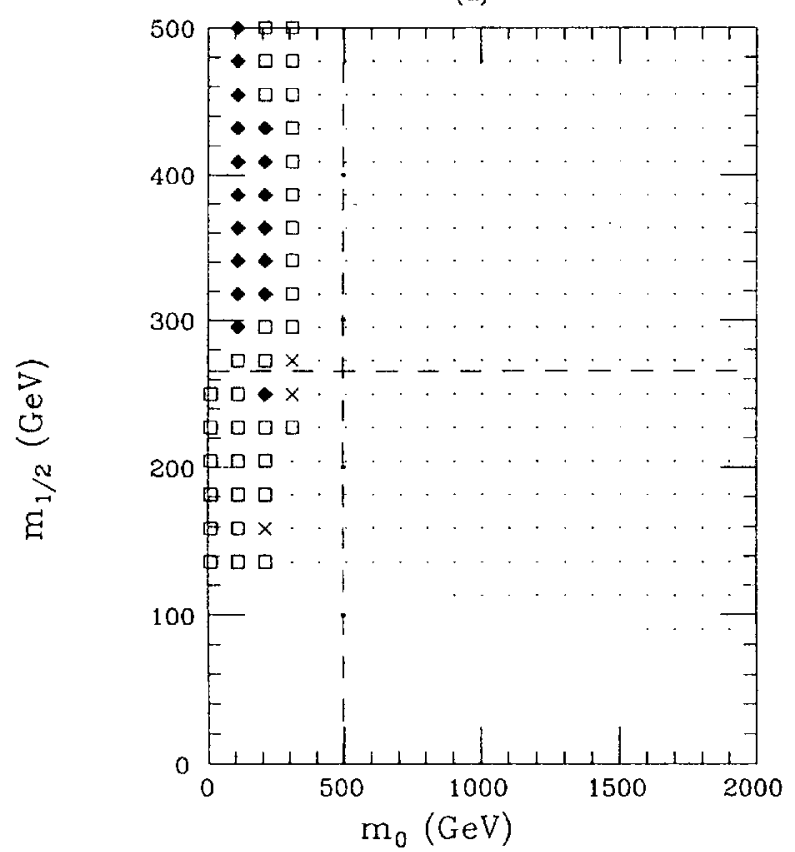

(b)

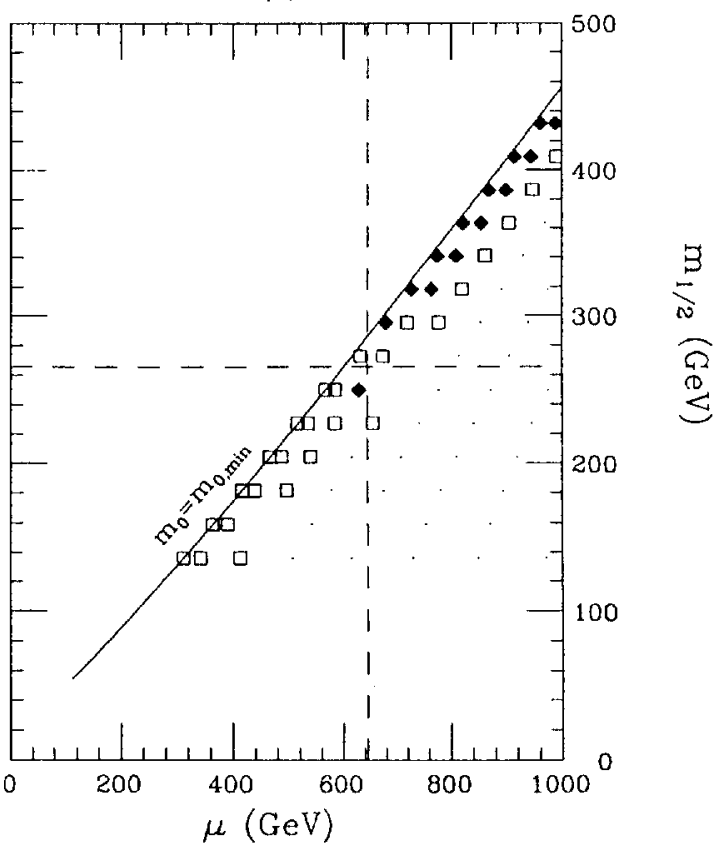

Fig. 2. (a) The parameter space in the $\left(m_{1 / 2}, m_{0}\right)$ plane for $\tan \beta=1.5, \delta_{1}=0$ and $\delta_{2}=0, \alpha_{s}=0.1127$. The empty regions are excluded by: (i) accelerator constraints, (ii) radiative EWSB conditions, (iii) the LSP not being a neutralino. Dots represent the region where $\Omega_{x} h^{2}>1$. Regions with crosses are excluded by $b \rightarrow s \gamma$ and $m_{b}$ constraints. In the regions denoted by filled diamonds, $0.1 \leq \Omega_{\chi} h^{2} \leq 0.3$. (b) The parameter space represented in the $\left(m_{1 / 2}, \mu\right)$ plane. The line $m_{0}=m_{0, \mathrm{~min}}$ corresponds to the minimum value of $m_{0}$. Notations are the same as in a), but crosses are omitted here. The dashed lines denote the no-fine-tuning upper bounds on $m_{0}, m_{1 / 2}$ and $\mu$. 
(a)

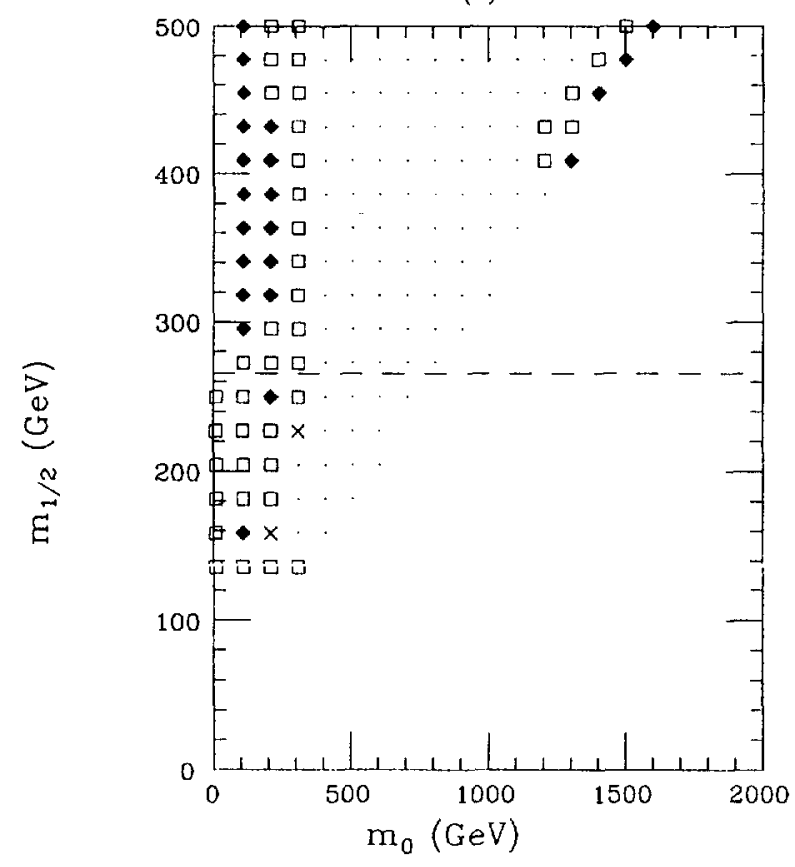

(b)

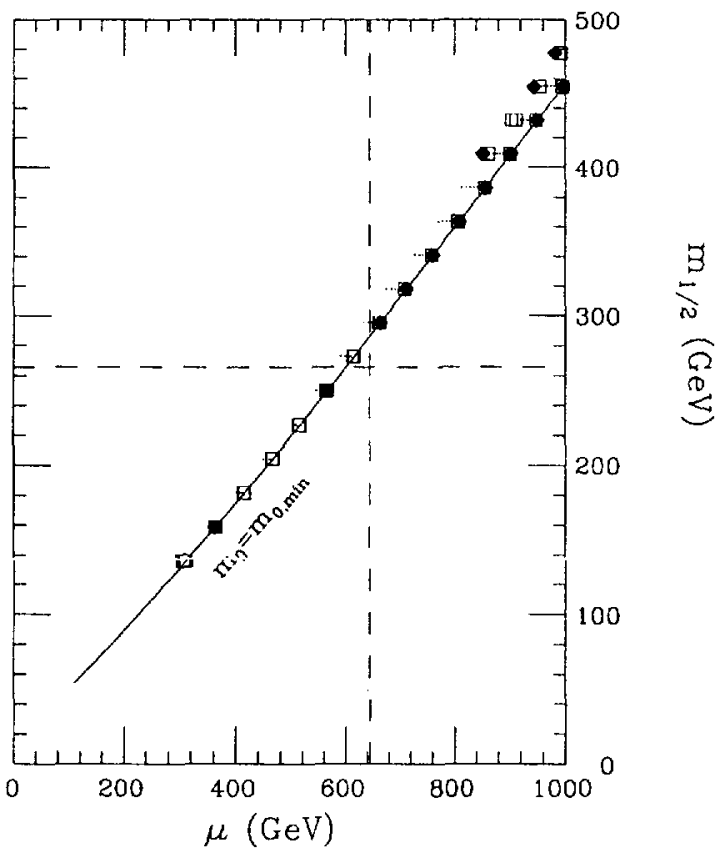

Fig. 3. The same as in Fig. 2, but with $\delta_{1}=-1.0$ and $\delta_{2}=1.0$.

(a)

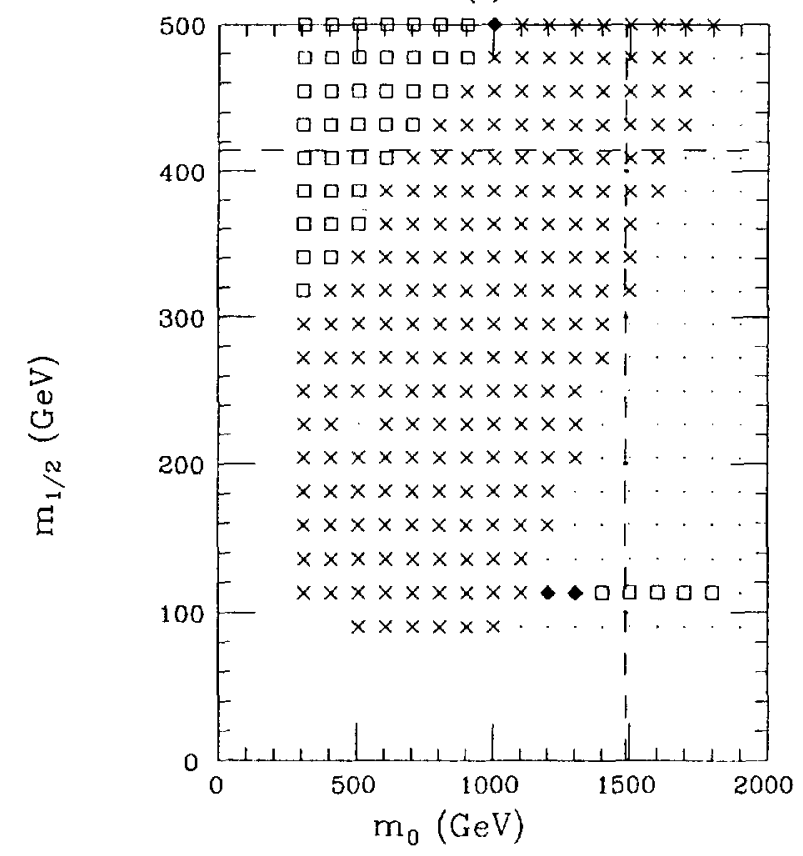

(b)

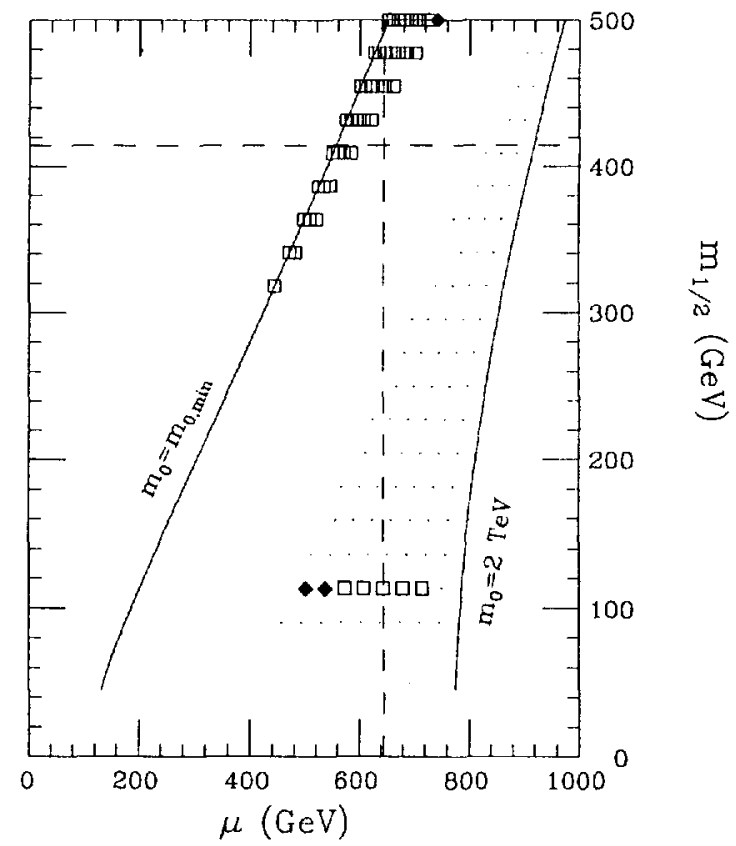

Fig. 4. The same as in Fig. 2, but with $\tan \beta=53, \delta_{1}=0.4$ and $\delta_{2}=-0.1 . \alpha_{s}=0.1127$. 
cal bound $\Omega h^{2} \leq 1$. The allowed region (denoted by squares) is mainly due to configurations where $\sigma_{\mathrm{ann}}$ is dominated by $\tilde{l}$ exchange. Filled diamonds denote configurations where $\Omega_{\chi} h^{2}$ is in the range of Eq. (4). In these configurations typical values for the mass parameters are: $m_{\tilde{l}} \simeq 150-200 \mathrm{GeV}, m_{\tilde{q}} \geq 600$ $\mathrm{GeV}, M_{A} \simeq 1 \mathrm{TeV}$. In Fig. 2(b) we notice that the allowed domain extends to the right of the line $m_{0}=m_{0, \min }$ (where $m_{0, \min }$ is the minimal value for $m_{0}$ ), allowing for the neutralino only a gauginodominated region. This occurs because the coefficient $J_{2}$ of Eq. (6) is positive (see Fig. 1).

An illustration of how a deviation from $m_{0}$ universality may somewhat modify the picture is provided by Fig. 3, where we display the relevant physical regions when we choose a departure from universality: $\delta_{1}=-1.0, \delta_{2}=1.0$, which makes the coefficient $J_{2}$ very small and negative. As a consequence, $m_{1 / 2}$ and $\mu$ are strongly correlated, with a slight extension of the neutralino physical region toward the sector of higgsino-gaugino mixture for $m_{1 / 2} \gtrsim 400 \mathrm{GeV}$ (see Fig. 3(b)). These new configurations, which turn out to correspond to high values of $m_{0}: m_{0} \simeq 1.3-1.6 \mathrm{TeV}$, are no longer in conflict with the cosmological bound, at variance with the universal case. In these points of the parameter space $\sigma_{\text {ann }}$ is dominated by the $\tilde{t}$-exchange, since here $m_{\tilde{i}} \simeq 250 \mathrm{GeV}$. However, we remark that these configurations realize radiative EWSB only with strong fine tuning. The generic trend is to have, for $\delta_{1}=$ $-1.0, \delta_{2}=1.0, \Omega_{\chi} h^{2}$ smaller than in the universal case and this feature is displayed in Fig. 3, where we notice that the cosmological constraint is less effective here in restricting the parameter space, as compared to the universal case (see Fig. 2).

Let us turn now to large values of $\tan \beta$. As was already pointed out in Ref. [6] for the universal case at $\tan \beta=53$, the neutralino relic abundance is very low: $\Omega_{\chi} h^{2} \leq 0.1$, due to small values of $M_{A}: M_{A} \leqslant$ $150 \mathrm{GeV}$, which generates a large $\sigma_{\text {ann }}$ dominated by Higgs-exchange contributions.

By moving away from $m_{0}$ universality, we can change the picture noticeably. For instance, by taking $\delta_{1}=0.4, \delta_{2}=-0.1$, we can generate large values of $\Omega_{\chi} h^{2}$. However, as we see in Fig. 4, most of the configurations of large $\Omega_{\chi} h^{2}$ are disallowed by the constraint on the bottom mass (and also marginally by the constraint $b \rightarrow s y$ ). The reason for (a)

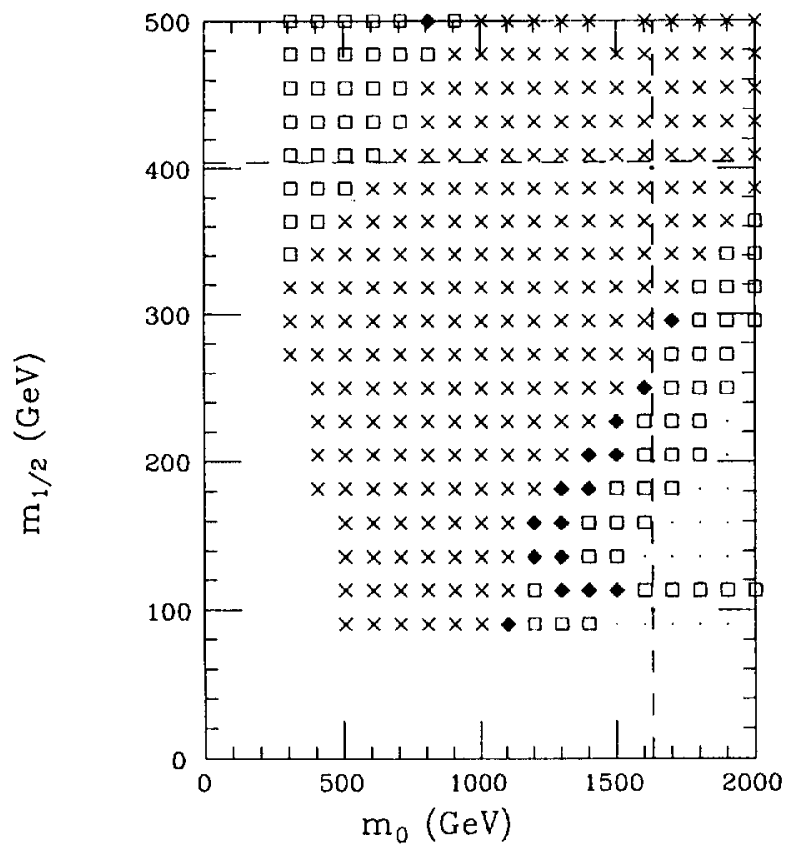

(b)

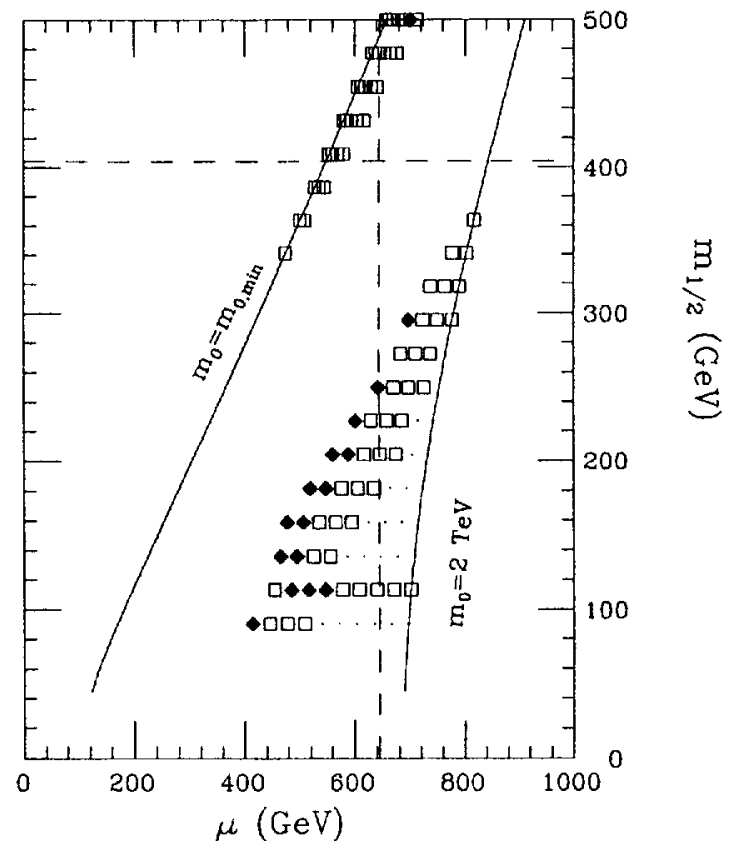

Fig. 5. The same as in Fig. 4, but with $\alpha=0.118$. 
the marked difference between this case and the universal one is that now $M_{A}$ is large. Indeed, the lowest allowed values of $M_{A}\left(M_{A} \approx 150 \mathrm{GeV}\right)$ only occur in the parameter region: $m_{0} \simeq 300 \mathrm{GeV}, m_{1 / 2}$ $\simeq 400 \mathrm{GeV}$. The large values of $M_{A}$ are caused by a sizeable, positive value for the coefficient $K_{2}$ of Eq. (7). The only allowed configurations which provide $\Omega_{\chi} h^{2}$ in the range of Eq. (4) are those with $m_{1 / 2} \simeq$ $120 \mathrm{GeV}$ and $1.2 \mathrm{TeV} \leq m_{0} \leq 1.4 \mathrm{TeV}$. Notice that some of these configurations do not satisfy our nofine-tuning constraint. The relevance of the $\alpha_{s}$ value in determining $\Omega_{\chi} h^{2}$ and in shaping the allowed physical regions is apparent when Fig. 4, evaluated for $\alpha_{s}=0.1127$, is compared to Fig. 5, evaluated for $\alpha_{s}=0.118$.

\section{Evaluation of the signals}

As was anticipated in the Introduction, the signals to be discussed in the present paper consist of the fluxes of up-going muons through a neutrino telescope generated by neutrinos which are produced by pair annihilations of neutralinos captured and accumulated inside the Earth and the Sun. The steps involved in this process are the following: (a) capture by the celestial body of the relic neutralinos through a slow-down process due essentially to neutralino elastic scattering off the nuclei of the macroscopic body, (b) accumulation of the captured neutralinos in the central part of the celestial body, (c) neutralino-neutralino annihilation with emission of neutrinos, and for the various annihilation products, (d) propagation of neutrinos and conversion of their $\nu_{\mu}$ component into muons in the rock surrounding the detector (or, much less efficiently, inside the detector), and finally (e) propagation and detection of the ensuing up-going muons in the detector.

The various quantities relevant for the previous steps are calculated here according to the method described in Ref. [8], to which we refer for further details.

\subsection{Neutralino local density}

As will be shown explicitly in the subsequent subsection, the capture rate of the relic neutralinos is proportional to the local neutralino density $\rho_{x}$ in the solar neighbourhood. Let us specify here how $\rho_{\chi}$ is evaluated.

For each point of the model parameter space we first calculate the relevant value of the cosmological neutralino relic density according to the procedure outlined in Section 4. Whenever $\Omega_{\chi} h^{2}$ is larger than a minimal $\left(\Omega h^{2}\right)_{\min }$ suggested by observational data and by large scale structure calculations, we simply put $\rho_{\chi}=\rho_{1}$, where $\rho_{1}$ is the local halo density. In the points of the parameter space where $\Omega_{\chi} h^{2}$ is less than $\left(\Omega h^{2}\right)_{\min }$, the neutralino may only provide a fractional contribution $\Omega_{\chi} h^{2} /\left(\Omega h^{2}\right)_{\min } \equiv \xi$ to $\Omega h^{2}$; in this case we take $\rho_{\chi}=\rho_{1} \xi$. The value to be assigned to $\left(\Omega h^{2}\right)_{\min }$ is somewhat arbitrary. Here it is set equal to 0.1 . As far as the local halo density $\rho_{1}$ is concerned, we are inspired by the recent estimate $\rho_{1}=0.51_{-0.17}^{+0.21} \mathrm{GeV} \mathrm{cm}^{-3}$ [38], based on a flattened dark matter distribution and recent microlensing data. This introduces a significantly larger central value as compared to previous determinations (see, for instance, Ref. [39]). All the numerical results presented in this paper were obtained using the value $\rho_{1}=0.5$ $\mathrm{GeV} \mathrm{cm}^{-3}$.

\subsection{Capture rates and annihilation rates}

For the evaluation of the capture rate $C$ of the relic neutralinos by a celestial body we have used the standard formula [40]

$C=\frac{\rho_{\chi}}{v_{\chi}} \sum_{i} \frac{\sigma_{i}}{m_{\chi} m_{i}}\left(M_{\mathrm{B}} f_{i}\right)\left\langle v_{\mathrm{esc}}^{2}\right\rangle X_{i}$,

where $v_{x}$ is the neutralino mean velocity, $\sigma_{i}$ is the cross section of the neutralino elastic scattering off the nucleus $i$ of mass $m_{i}, M_{\mathrm{B}} f_{i}$ is the total mass of the element $i$ in the body of mass $M_{\mathrm{B}},\left\langle v_{\mathrm{esc}}^{2}\right\rangle_{i}$ is the square escape velocity averaged over the distribution of the element $i$, and $X_{i}$ is a factor which takes account of kinematical properties occurring in the neutralino-nucleus interactions. For the evaluation of the elastic $\chi$-nucleus cross sections we refer to [6].

The annihilation rate $\Gamma_{A}$ of the neutralinos inside the macroscopic body is calculated with the formula [41]

$\Gamma_{A}=\frac{C}{2} \tanh ^{2}\left(\frac{t}{\tau_{A}}\right)$ 
where $t$ is the age of the macroscopic body $(t=4.5$ Gyr for Sun and Earth), $\tau_{A}=\left(C C_{A}\right)^{-1 / 2}$, and $C_{A}$ is the annihilation rate per effective volume of the body, given by

$C_{A}=\frac{\langle\sigma v\rangle}{V_{0}}\left(\frac{m_{\chi}}{20 \mathrm{GeV}}\right)^{3 / 2}$

Here, $V_{0}$ is defined as $V_{0}=\left(3 m_{\mathrm{Pl}}^{2} T /(2 \rho \times 10\right.$ $\mathrm{GeV}))^{3 / 2}$ where $T$ and $\rho$ are the central temperature and the central density of the celestial body. For the Earth $\left(T=6000 \mathrm{~K}, \rho=13 \mathrm{~g} \mathrm{~cm}^{-3}\right) V_{0}=2.3 \times 10^{25}$ $\mathrm{cm}^{3}$, for the Sun $\left(T=1.4 \times 10^{7} \mathrm{~K}, \rho=150 \mathrm{~g} \mathrm{~cm}^{-3}\right)$ $V_{0}=6.6 \times 10^{28} \mathrm{~cm}^{3}$. Also, $\sigma_{\mathrm{ann}}$ is the neutralinoneutralino annihilation cross section and $v$ is the relative velocity: $\left\langle\sigma_{\mathrm{ann}} v\right\rangle$ is calculated with all the contributions at the tree level as previously discussed in Section 4, with the further inclusion here of the two-gluon annihilation final state [42].

From Eq. (9) it follows that in a given macroscopic body the equilibrium between capture and annihilation (i.e. $\Gamma_{A} \sim C / 2$ ) is established only when $t \geq \tau_{A}$. We stress here that the neutralino density $\rho_{X}$ enters not only in $C$ but also in $\tau_{A}$ (through $C$ ). Thus, the use of a correct value for $\rho_{x}$ (rescaled, when necessary) is important also in determining whether or not equilibrium is reached in a macroscopic body.

From the evaluation of the annihilation rate for neutralinos inside the Earth and the Sun it turns out that, for the Earth, the equilibrium condition depends sensitively on the values of the model parameters, and is not satisfied in wide regions of the parameter space. Consequently, for these regions the signal due to neutralino annihilation may be significantly attenuated. On the contrary, in the case of the Sun, equilibrium between capture and annihilation is reached for the whole range of $m_{\chi}$, due to the much more efficient capture rate due to the stronger gravitational field [40].

\subsection{Neutrino fluxes}

Let us turn now to the evaluation of the neutrino fluxes due to the annihilation processes taking place in the celestial bodies. For a distant source such as the Sun the differential rate in the neutrino energy $E_{\nu}$ is given by

$\frac{\mathrm{d} N_{v}}{\mathrm{~d} E_{\nu}}=\frac{\Gamma_{A}}{4 \pi d^{2}} \sum_{F, f} B_{\chi f}^{(F)} \frac{\mathrm{d} N_{f v}}{\mathrm{~d} E_{\nu}}$

where $d$ is the distance from the source, $F$ denotes the $\chi-\chi$ annihilation final states, $B_{\chi f}^{(F)}$ denotes the branching ratios into heavy quarks, $\tau$ leptons and gluons in the channel $F ; \mathrm{d} N_{f \nu} / \mathrm{d} E_{\nu}$ is the differential distribution of the neutrinos generated by the hadronization of quarks and gluons and the subsequent hadronic semileptonic decays.

In the case of the Earth one has to take into account the size of the region around the center of the Earth where most of the neutralinos are accumulated. This is important, since the angular dependence of the flux plays a crucial role in providing a signature, and, potentially, in allowing a determination of the neutralino mass $[8,40,43]$. For details about the relevant formulae we refer to [8]. Here we only give some information about our evaluation of the spectrum $\mathrm{d} N_{f v} / \mathrm{d} E_{v}$ to be employed in Eq. (11). The neutrino spectra due to $b$ and $c$ quarks, $\tau$ leptons and gluons were computed using the Jetset 7.2 Monte Carlo code [44]. We have neglected the contributions of the light quarks directly produced in the annihilation process or in the hadronization of heavy quarks and gluons, because these light particles stop inside the medium (Sun or Earth) before their decay [45]. For the case of the Sun we have also considered the energy loss of the heavy hadrons in the solar medium. The spectra due to heavier final states, i.e. Higgs bosons, gauge bosons and $\mathrm{t}$ quark, were computed analytically by following the decay chain down to the production of $\mathrm{a} b$ quark, $\mathrm{c}$ quark or a tau lepton; the result of the Monte Carlo was used to obtain the final neutrino output ${ }^{10}$. Because of the high column density of the solar medium, the absorption and the energy loss of the produced neutrinos were also included.

One possible effect that we have not included is that of matter-enhanced neutrino oscillations. As pointed out in Ref. [47], these could be important for neutrinos produced by neutralino annihilations in the Sun, if there is a large mixing angle as in some fits

\footnotetext{
${ }^{10}$ For a semi-analytic approach, alternative to the Monte Carlo calculation employed in the present paper, see Ref. [46].
} 
to solar or atmospheric neutrino data. It would have been more conservative to allow for $\nu_{\mu} \rightarrow \nu_{\mathrm{e}}$ or $\nu_{\tau}$ oscillations inside the Sun, but we neglect them here.

\subsection{Fluxes of up-going muons}

The capability of a neutrino telescope to measure the flux of Eq. (11) depends on how well this signal may be discriminated from the background due to neutrinos produced in the Earth's atmosphere by cosmic rays. In the case of the Sun, the signal to background (S/B) discrimination is based on the correlation with the position of the Sun in the sky. As far as the signal from the Earth is concerned, the $\mathrm{S} / \mathrm{B}$ discrimination requires an analysis of the angular distribution, and is based on the property that, whereas the angular distribution of the signal is expected to be markedly peaked at the nadir, the background distribution is almost flat (with a slight increase at the horizon). In order to evaluate these angular distributions properly, one has to take into account the size of the region around the center of the Earth where neutralinos are expected to accumulate, and the physical processes that entail an angular spreading of the signal. Some angular spreading is induced by the $\nu_{\mu} \rightarrow \mu$ conversion: $\theta_{\mu, \nu} \simeq 2^{\circ}\left(E_{\nu} / 100\right.$ $\mathrm{GeV})^{1 / 2}$, and a comparable effect occurs because of the multiple scattering of the muon in the rock surrounding the detector. Thus, an accurate calculation of angular distributions requires a Monte Carlo simulation [8].

In this paper, since we only deal with spectra, and not with angular distributions, we perform the calculation of the up-going muon fluxes in a simple no-straggling approximation [48]. In fact, this approximation reproduces accurately the Monte Carlo calculations for the distributions in the muon energy [8]. Then, we can write the muon spectrum as [48]

$$
\begin{aligned}
\frac{\mathrm{d} N_{\mu}}{\mathrm{d} E_{\mu}}= & N_{A} \frac{1}{A+B E_{\mu}} \\
& \times \int_{E_{\mu, \text { th }}}^{\infty} \mathrm{d} E_{\nu} \frac{\mathrm{d} N_{\nu}}{\mathrm{d} E_{\nu}} \int_{E_{\mu}}^{E_{\nu}} \mathrm{d} E_{\mu}^{\prime} \frac{\mathrm{d} \sigma\left(E_{\nu}, E_{\mu}^{\prime}\right)}{\mathrm{d} E_{\mu}^{\prime}}
\end{aligned}
$$

where $E_{\mu, \text { th }}$ is the muon energy threshold, $N_{A}$ is the Avogadro number, $\mathrm{d} \sigma\left(E_{\nu}, E_{\mu}^{\prime}\right) / \mathrm{d} E_{\mu}^{\prime}$ is the differential cross section for the production of a $\mu$ from a $\nu_{\mu}$ impinging on an isoscalar target and $A+B E_{\mu}$ is the average muon energy loss, due to ionization, pair production, bremsstrahlung and photonuclear effects. For the coefficients $A$ and $B$ we have used the following values $A=2.4 \cdot 10^{-3} \mathrm{GeV} /\left(\mathrm{g} / \mathrm{cm}^{2}\right), B$ $=4.75 \cdot 10^{-6}\left(\mathrm{~g} / \mathrm{cm}^{2}\right)^{-1}[49]$.

Let us conclude this section with some comments on the typical parameters of the required experimental layout. In deep underwater/ice experiments muons from neutralino annihilation are planned to be detected using their direct Cerenkov radiation. A relativistic muon in water emits per $1 \mathrm{~cm}$ about 250 Cerenkov photons with wavelengths in the interval $300-500 \mathrm{~nm}$. Then it is easy to estimate the number of photoelectrons produced in a photomultipler (PM) at a distance $r$ from muon trajectory as

$N_{\mathrm{e}}=30 \frac{Q}{r} D_{\mathrm{PM}}^{2}$

where $Q \simeq 0.15-0.25$ is the PM quantum efficiency, all distances are given in $\mathrm{cm}$ and we take $D_{\mathrm{PM}} \simeq 35$ $\mathrm{cm}$ as the PM diameter. One can see from Eq. (13) that at a distance equal to the scattering length in the water/ice, which we take as $l_{\mathrm{sc}} \sim 20-30 \mathrm{~m}$, PM's detect a strong signal corresponding to about 3 photoelectrons. To determine the muon trajectory, only the PM's located closer than the scattering length can be used. Their number, $N_{\mathrm{PM}} \simeq l_{\mu} l_{\mathrm{sc}}^{2} / d^{3}$, is of order $100\left(E_{\mu} /(100 \mathrm{GeV})\right)$ for a distance between detectors $d=10 \mathrm{~m}$ and a scattering length $25 \mathrm{~m}$.

The supersymmetric model we are discussing is characterized by light neutralinos, $m_{\chi} \lesssim 200 \mathrm{GeV}$. This results in a small pathlength of the produced muons, $l_{\mu}=500\left(E_{\mu} /(100 \mathrm{GeV})\right) \mathrm{m}$, which has the following observational consequences.

- For a widely-discussed $1 \mathrm{~km}^{3}$ array, many muons have trajectories confined within the detector. The energies of these muons can be estimated from the trajectory lengths.

- There is a small probability to find a big shower due to bremsstrahlung or pair production along the muon trajectory.

- A reliable measurement of the muon trajectory, which is necessary for identification of a neutralino-produced muon, needs a rather dense array with a distance between detectors $d \sim 10 \mathrm{~m}$ in the case of a scattering length $25-30 \mathrm{~m}$. It could be the core of a larger detector. 


\section{Results and conclusions}

In this section we show our results for integrated fluxes $\Phi_{\mu}$ of the up-going muons, and compare them with the upper bounds obtained at the present neutrino telescopes. We will then compare these indirect signals with the signals measurable by direct searches for relic neutralinos. We also show how the strengths of the various signals correlate with each other and with the values of the neutralino relic abundance. However, we wish first to comment on some general properties which later provide a simple interpretation of some characteristic features of our numerical results.

(i) Both the event rate for neutralino direct detection $R$ and the capture rate $C$ of a relic neutralino by a celestial body are proportional to $\rho_{\chi} \sigma_{i}$. This property also applies to $\Phi_{\mu}$, which, however, has some further dependence on $\sigma_{i}$ and $\sigma_{\mathrm{ann}}$ through $\tau_{A}$. Thus, we expect $R$ and $\Phi_{\mu}$ to show some similarities in their behaviours as functions of the supersymmet- ric parameters, when the same elastic cross sections $\sigma_{i}$ 's are involved. This is roughly the case, since neutralino capture in a macroscopic body occurs dominantly (except for extremely pure gaugino compositions of the neutralino) through coherent cross sections, which have an overwhelming role also in direct detection by the nuclei considered here. Some deviations from such behaviours occur in two instances: (a) for $\Phi_{\mu}^{\text {Earth }}$, when equilibrium between capture and annihilation in the Earth is not yet reached (because of the extra dependence (mentioned above) of $\Phi_{\mu}^{\text {Earth }}$ on $\sigma_{i}$ and $\sigma_{\text {ann }}$ through $\tau_{A}$ ),

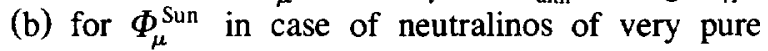
gaugino compositions, since these neutralinos are captured mainly through spin-dependent cross sections for scattering on hydrogen nuclei in the Sun.

(ii) If we combine the properties: $R \propto \rho_{1} \sigma_{i}, \Phi_{\mu} \propto$ $\rho_{1} \sigma_{i}$, and $\Omega_{\chi} h^{2} \propto\left\langle\sigma_{\mathrm{ann}} v\right\rangle_{\text {int }}^{-1}$, and take into account the fact that usually $\sigma_{i}$ and $\sigma_{\mathrm{ann}}$, as functions of the supersymmetric model parameters, are either both increasing or both decreasing, we come to the con- (a)

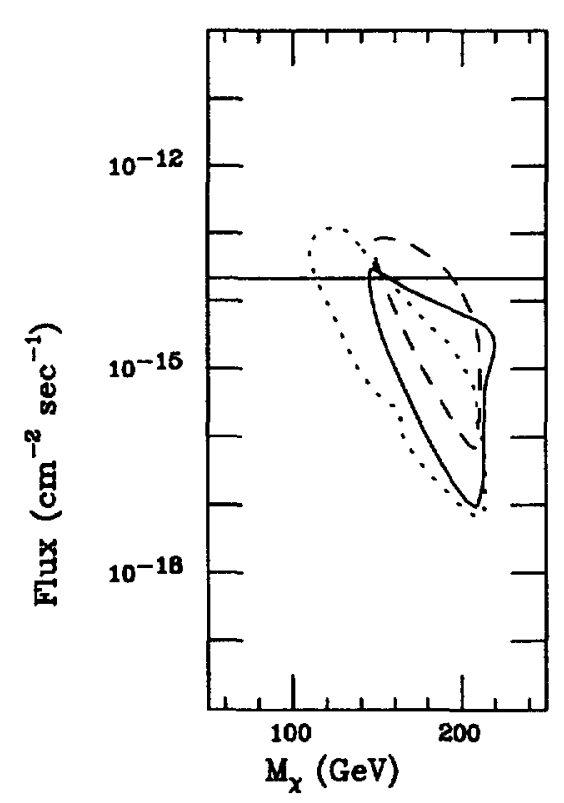

(b)

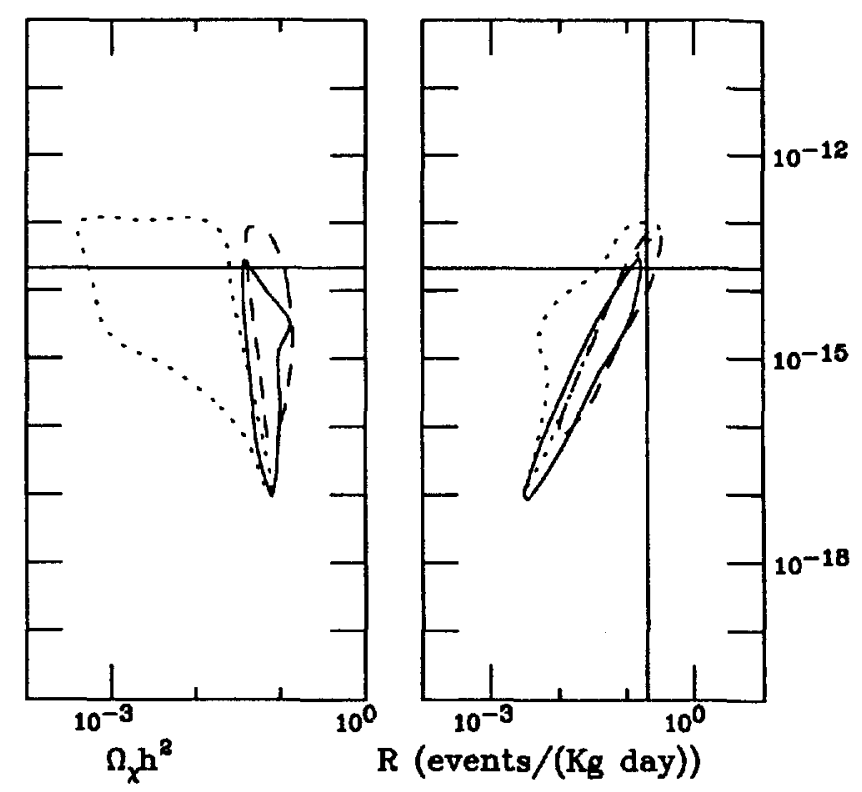

Fig. 6. The flux $\Phi_{\mu}^{\text {Earth }}$ for $\tan \beta=53: \delta_{1}=0, \delta_{2}=0$ and $\alpha_{s}=0.1127$ (solid line), $\delta_{1}=0, \delta_{2}=-0.3$ and $\alpha_{s}=0.118$ (dashed line), and $\delta_{1}=0.7, \delta_{2}=0.4$ and $\alpha_{s}=0.115$ (dotted line) as a function of $m_{\chi}$ (a), as a function of $\Omega_{\chi} h^{2}$ (b), and plotted versus the rate for direct detection with a Ge detector (c). Allowed configurations stay inside the closed curves. The horizontal line displays the experimental bound $\Phi_{\mu}^{\text {Earth }} \leq 2.1 \cdot 10^{-14} \mathrm{~cm}^{-2} \mathrm{~s}^{-1}(90 \%$ C.L.) [15], and the vertical line of section (c) displays the upper limit of Ref. [54]. Parameters are varied on a linear equally-spaced grid over the ranges: $10 \mathrm{GeV} \leq m_{0} \leq 2 \mathrm{TeV}, 45 \mathrm{GeV} \leq m_{1 / 2} \leq 500 \mathrm{GeV}$. 
clusion that the quantities $\Phi_{\mu}$ and $R$ are somewhat anticorrelated with $\Omega_{\chi} h^{2}$ (see also [50]). This property is attenuated, but usually not washed out, in the case of scaling of the neutralino local density. In fact, when scaling occurs (i.e., when $\Omega_{\chi} h^{2}<$ $\left.\left(\Omega h^{2}\right)_{\min }\right)$, one has $R \propto \rho_{1} \xi \sigma_{i} \propto \rho_{l} \sigma_{i} \times$ $\left[\left\langle\sigma_{\mathrm{ann}} v\right\rangle_{\mathrm{int}}\right]^{-1}$, and the same for $\Phi_{\mu}$. Since it turns out that when $\sigma_{i}$ is large also $\sigma_{\text {ann }}$ increases, but in such a way that usually the ratio $\sigma_{i} / \sigma_{\text {ann }}$ increases too, one can conclude that a form of anticorrelation between $\Omega_{\chi} h^{2}$ and $\Phi_{\mu}$ (or $R$ ) persists also when scaling of $\rho_{1}$ is effective. A feature of this type is displayed by our numerical results.

Let us turn now to a presentation of some of our results. We show in Fig. 6 a sample of our calculations for the signals expected from the Earth at the representative point $\tan \beta=53$, for three different choices of the $\delta_{i}$ parameters. The three closed curves denote the boundaries of the allowed regions when the parameters $m_{0}$ and $m_{1 / 2}$ are varied in the ranges
$10 \mathrm{GeV} \leq m_{0} \leq 2 \mathrm{TeV}, 45 \mathrm{GeV} \leq m_{1 / 2} \leq 500 \mathrm{GeV}$. The figure displays the flux of upgoing muons integrated over a cone of half aperture of $30^{\circ}$ centered at the nadir, and for muon energies above $1 \mathrm{GeV}$. Also shown in Fig. 6 is the most stringent experimental bound: $\Phi_{\mu}^{\text {Earth }} \leq 2.1 \cdot 10^{-14} \mathrm{~cm}^{-2} \mathrm{~s}^{-1}$ (90\% C.L.), obtained with an exposure of $2954 \mathrm{~m}^{2} \mathrm{yr}$ [16] (for other recent experimental data see [51-53]).

In Fig. 6(a) we notice, in the case $\delta_{1}=0, \delta_{2}=0$, $\alpha_{s}=0.1127$ indicated by the solid line, a decreasing behaviour of $\Phi_{\mu}$ as a function of the neutralino mass, as expected from the structure of the capture rate $C$. It is remarkable that for some neutralino configurations in the interesting range $m_{\chi} \simeq 150 \mathrm{GeV}$ the present experimental sensitivity (or a slight improvement of it) is already adequate for restricting the supersymmetric parameter space. These configurations have a light $A: M_{A} \leq 70 \mathrm{GeV}$. The anti-correlations between $\Phi_{\mu}$ and $\Omega_{\chi} h^{2}$ and the correlations between $\Phi_{\mu}$ and $R$, anticipated in points (i) and (ii) (a)

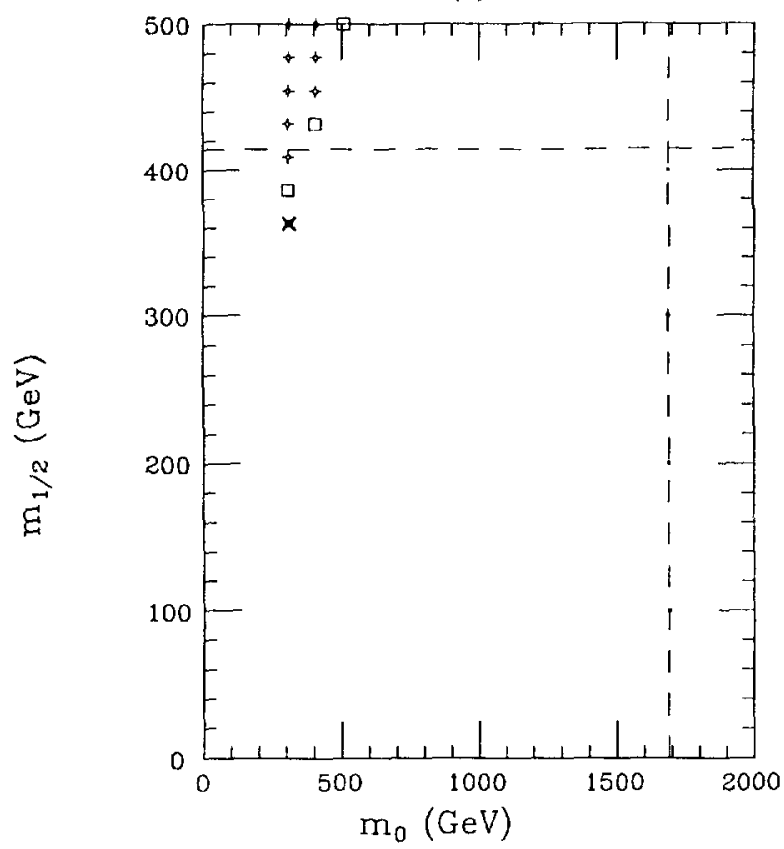

(b)

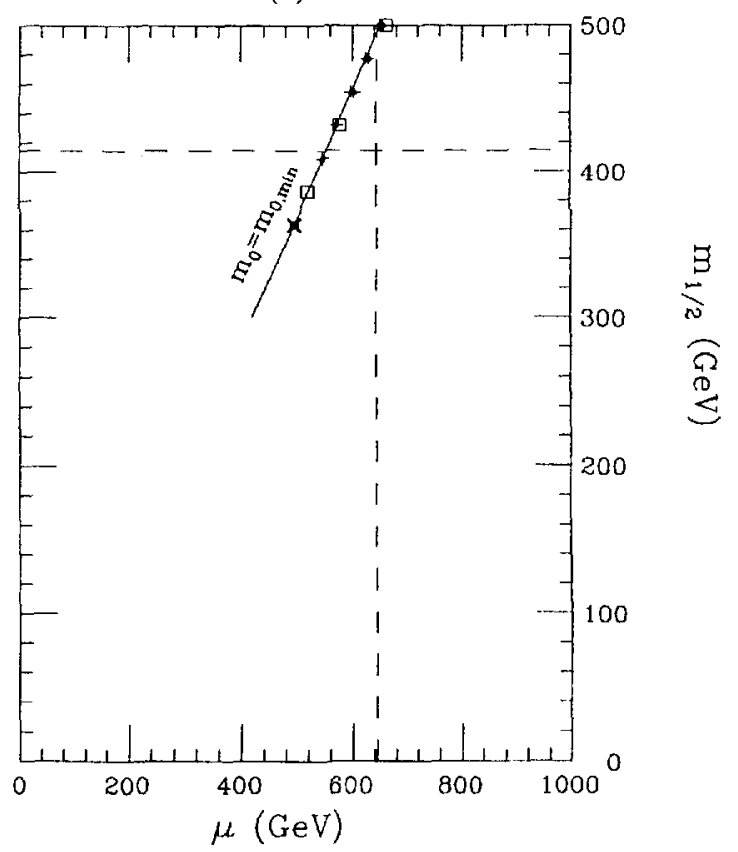

Fig. 7. (a) The parameter space in the $\left(m_{1 / 2}, m_{0}\right)$ plane for $\tan \beta=53, \delta_{1}=0$ and $\delta_{2}=0, \alpha_{s}=0.1127$. Empty regions are excluded by: (i) accelerator constraints, (ii) radiative EWSB conditions, (iii) the LSP not being a neutralino, (jv) $b \rightarrow s \gamma$ and $m_{b}$ constraints. The heavy oblique crosses denote configurations above the present experimental bound [16]. Squares denote regions which could be explored by a neutrino telescope whose sensitivity is one order of magnitude better than that of Ref. [16]. The region with light crosses would require an even better sensitivity. (b) The parameter space represented in the $\left(m_{1 / 2}, \mu\right)$ plane. 
above, are apparent in parts (b) and (c) of Fig. 6, respectively. One important feature of this parameter choice is shown by the solid line in Fig. 6(b): the neutralino configurations, which are explorable with measurements of $\Phi_{\mu}$, yield values of $\Omega_{\chi} h^{2}$ below the range of Eq. (4). We stress that this is a general trend, which will be further discussed at the end of this section. Fig. 6(c) shows how measurements of $\Phi_{\mu}$ and $R$ may give information about similar neutralino configurations. The event rate for direct detection $R$, employed in the present paper as well as in our previous work [6], is defined as the integral of $\mathrm{d} R / \mathrm{d} E_{\mathrm{ee}}$ ( $E_{\mathrm{ee}}$ is the electron equivalent energy) over the $12-13 \mathrm{keV}$ range, for a $\mathrm{Ge}$ detector. The experimental upper bound is that obtained from the experiment of Ref. [54] (for other experiments using Ge detectors see Ref. [55]).

The discovery potential of neutrino telescopes in this context is further illustrated in Fig. 7, where the case denoted by the solid line in Fig. 6 is shown. This figure displays (by empty squares) the region in the $m_{1 / 2}-m_{0}$ plot (and in the $m_{1 / 2}-\mu$ plot) which may be explored by a neutrino telescope whose sensitivity is one order of magnitude better than that of Ref. [16]. The region denoted by light crosses would require an even better sensitivity. In this connection, we stress that the theoretical results presented here were obtained using a particular set of representative values, not only for some supersymmetric model parameters, but also for a number of astrophysical and cosmological parameters, many of which are affected by large experimental uncertainties. This is the case, for instance, of the dark matter local density $\rho_{1}$ and of $\Omega h^{2}$. For this reason, one has to be extremely cautious in extracting exclusion plots in the supersymmetric parameters from a comparison between present experimental and theoretical values of $\Phi_{\mu}$. Obviously, a region of the neutralino parameter space would be excluded only if the theoretical values, obtained with the most conservative set of values for the free parameters, were above the experimental upper bound. This is certainly not the case with the present experimental limits. Particular attention has to be paid to the value of the neutralino local density $\rho_{\chi}$ to be used. Just employing $\rho_{\chi}=\rho_{1}$ everywhere, without making a consistency check (a)

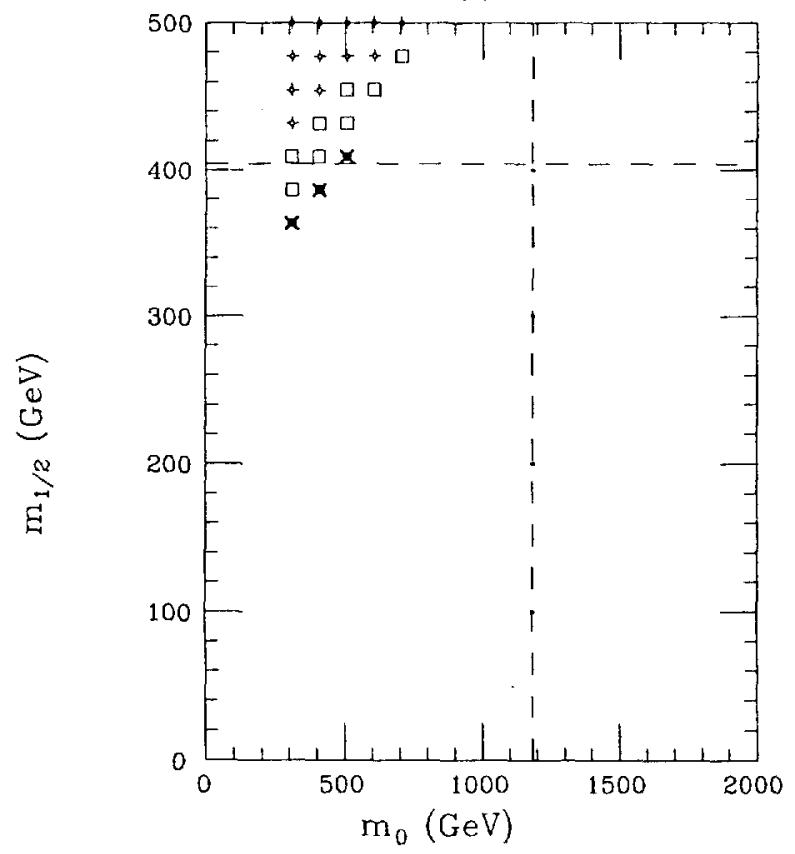

(b)

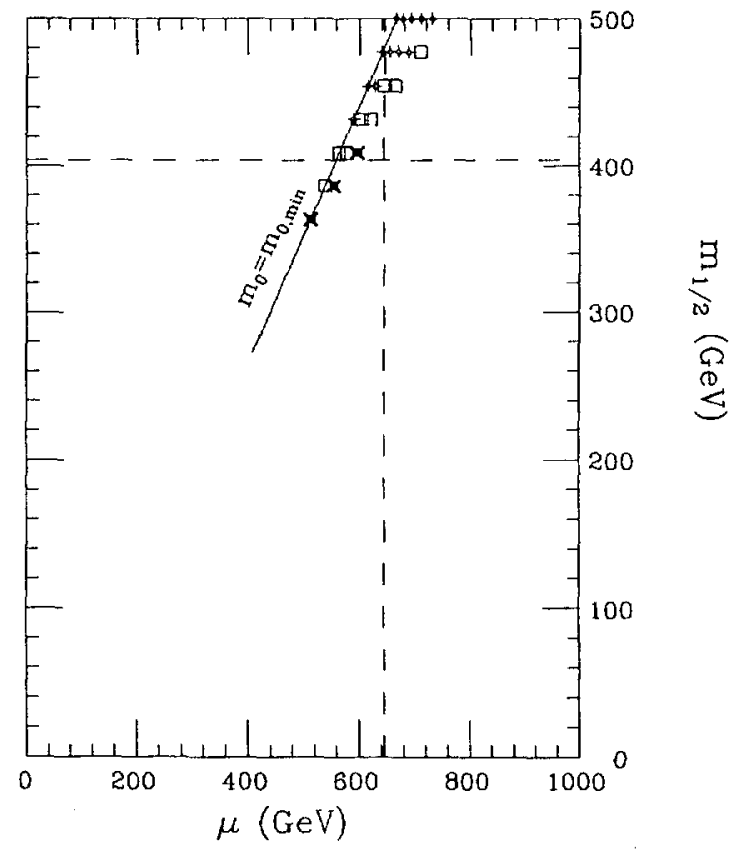

Fig. 8. The same as in Fig. 7, but with $\delta_{1}=0$ and $\delta_{2}=-0.3, \alpha_{s}=0.118$. 
(a)

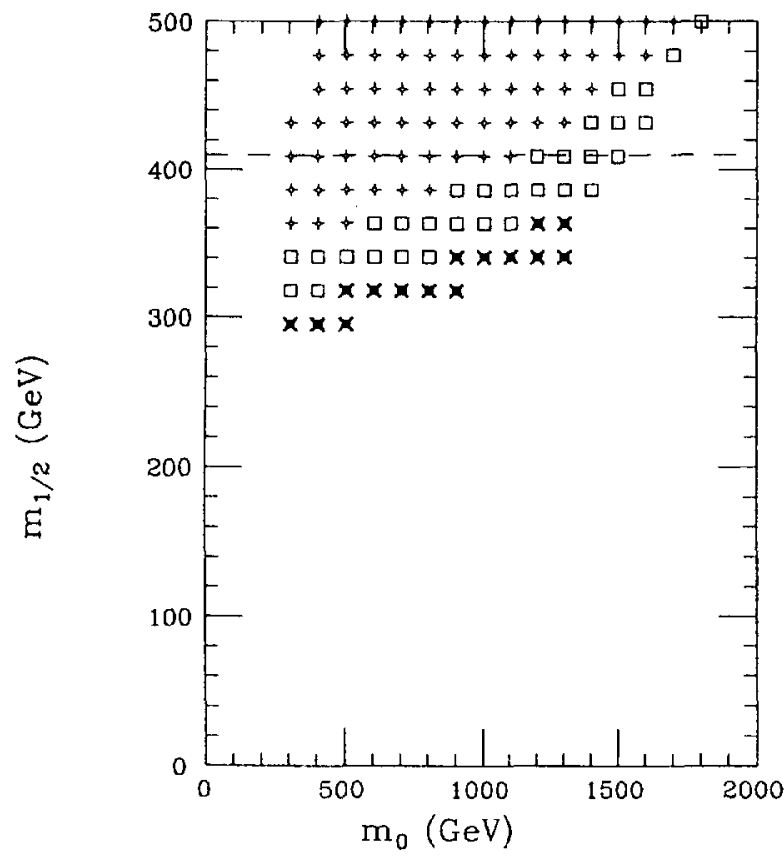

(b)

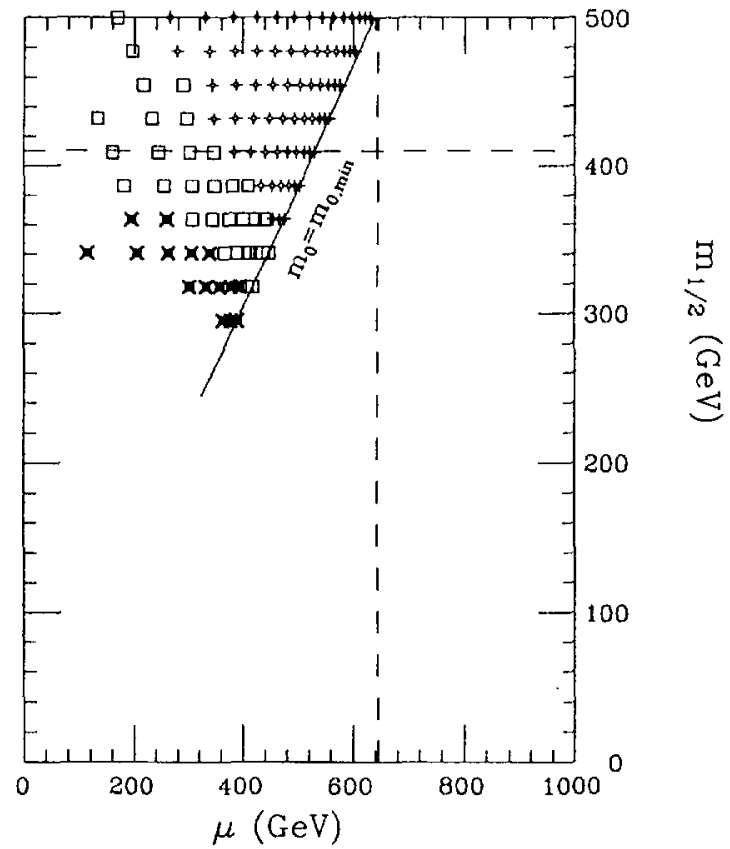

Fig. 9. The same as in Fig. 8, but with $\delta_{1}=0.7$ and $\delta_{2}=0.4, \alpha_{s}=0.115$.

(a)

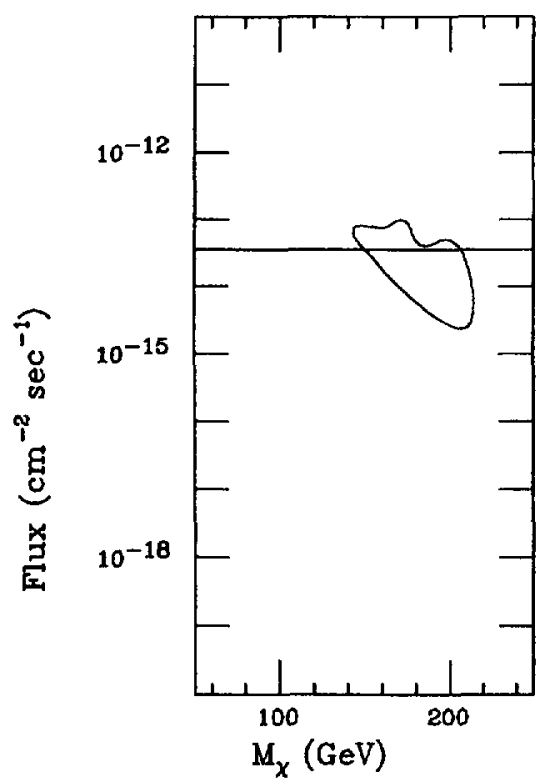

(b)

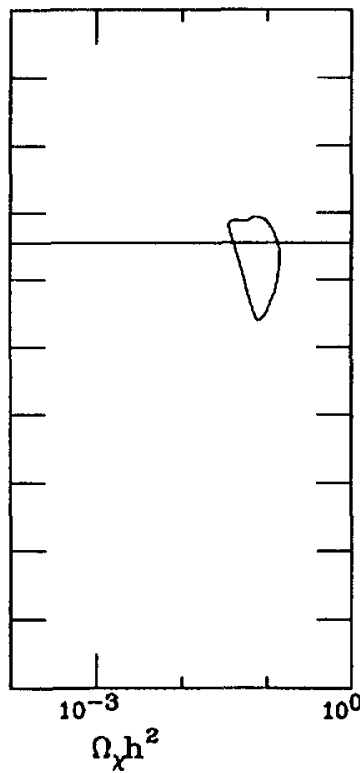

(c)

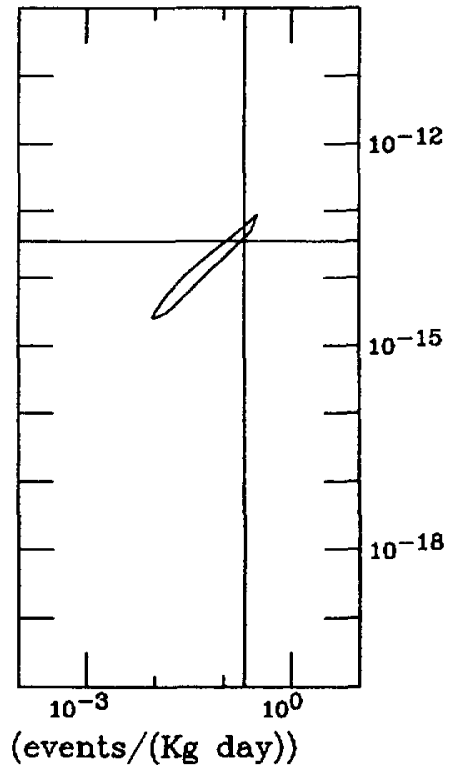

Fig. 10. The flux $\Phi_{\mu}^{\text {Sun }}$ for $\tan \beta=53, \delta_{1}=0$ and $\delta_{2}=-0.3, \alpha=0.118$, as a function of $m_{\chi}$ (a), as a function of $\Omega_{\chi} h^{2}$ (b), and plotted versus the rate for direct detection with a Ge detector (c). Allowed configurations stay inside the closed curves. The horizontal line displays the experimental bound $\Phi_{\mu}^{\text {Sun }} \leq 3.5 \cdot 10^{-14} \mathrm{~cm}^{-2} \mathrm{~s}^{-1}$ (90\% C.L.) [16], and the vertical line of section (c) displays the upper limit of Ref. [54]. Parameters are varied on a linear equally-spaced grid over the ranges: $10 \mathrm{GeV} \leq \mathrm{m}_{0} \leq 2 \mathrm{TeV}, 45 \mathrm{GeV} \leq m_{1 / 2} \leq 500 \mathrm{GeV}$. 
with the value of $\Omega_{\chi} h^{2}$ and without rescaling appropriately, leads to erroneous exclusion plots.

In Fig. 6 we present as dashed lines another set of results, corresponding to a case where a departure from universality in soft scalar masses is introduced: $\delta_{1}=0, \delta_{2}=-0.3$. We notice that many configurations may produce signals above the experimental bounds, both in direct and indirect searches. The dashed lines exhibit correlations between the various quantities similar to those exhibited by the solid lines, although in this case some configurations provide both a measurable $\Phi_{\mu}$ and an $\Omega_{\chi} h^{2}$ in the range of Eq. (4). The configurations whose signals are above the experimental limit are displayed in Fig. 8 with heavy oblique crosses. In particular, from Fig. 8(b) it is clear that the corresponding neutralino compositions are dominantly gaugino-like (notice that the allowed region in the $m_{1 / 2}-\mu$ plane extends to the right of the line $m_{0}=m_{0, \min }$, due to a positive value of the coefficient $J_{2}$ of Eq. (6)). It is worth recalling that the strict $m_{1 / 2}-\mu$ correlation appearing in Fig. 7 is removed here, due to the deviation from universality we have introduced (the coefficient $J_{2}$ is positive and sizeable here). The reason why many configurations are above the experimental bound, in spite of their gaugino nature, is the fact that in these configurations the CP-odd Higgs neutral boson $A$ is light: $m_{A} \leqq 65 \mathrm{GeV}$ (the coefficient $K_{2}$ of Eq. (7) is negative).

One further example of a nonuniversal case is provided by the dotted lines in Fig. 6 and by Fig. 9, where $\delta_{1}=0.7, \delta_{2}=0.4$. A number of configurations turn out again to exceed the experimental bound. Here the neutralino parameter space opens up to the left of the line $m_{0}=m_{0, \min }$, where gaugino-higgsino mixture takes place. Again, the configurations with the highest signals have a light $A$ boson.

Examples of signals from the Sun, $\Phi_{\mu}^{\text {Sun }}$, are (a)

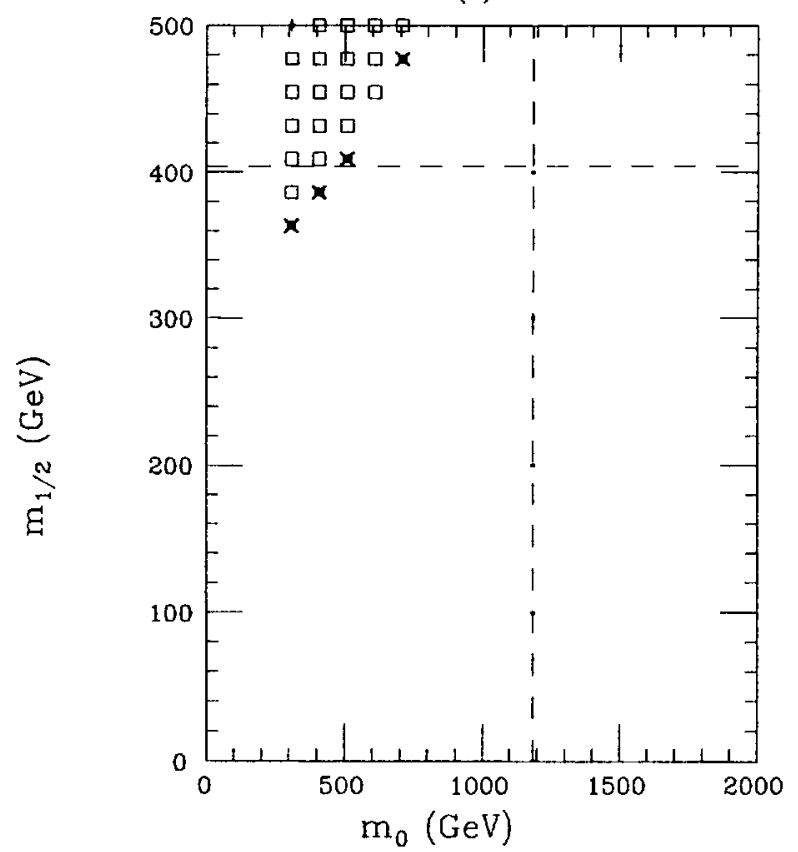

(b)

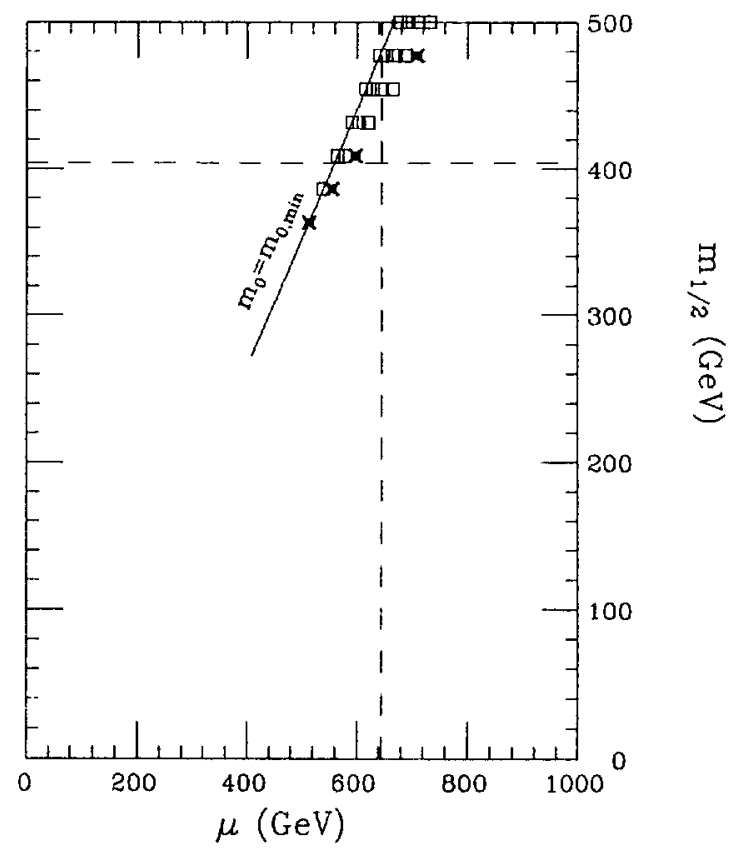

Fig. 11. (a) The parameter space in the $\left(m_{1 / 2}, m_{0}\right)$ plane for $\tan \beta=53, \delta_{1}=0$ and $\delta_{2}=-0.3, \alpha_{s}=0.118$. Empty regions are excluded by: (i) accelerator constraints, (ii) radiative EWSB conditions, (iii) the LSP not being a neutralino, (iv) $b \rightarrow s \gamma$ and $m_{b}$ constraints. The heavy oblique crosses denote configurations above the present experimental bound: $\Phi_{\mu}^{\text {Sun }} \leq 3.5 \cdot 10^{-14} \mathrm{~cm}^{-2} \mathrm{~s}^{-1}$ (90\% C.L.) [16]. Squares denote regions which could be explored by a neutrino telescope whose sensitivity is one order of magnitude better than that of Ref. [16]. The region with light crosses would require an even better sensitivity. (b) The parameter space represented in the $\left(m_{1 / 2}, \mu\right)$ plane. 
shown in Figs. 10 and 11 together with the value of the experimental bound: $\Phi_{\mu}^{\text {Sun }} \leq 3.5 \cdot 10^{-14} \mathrm{~cm}^{-2}$ $\mathrm{s}^{-1}$ (90\% C.L.), obtained with an exposure of 1002 $\mathrm{m}^{2} \mathrm{yr}[16]$. By comparing with Fig. 6, we notice that the distribution of points along the vertical axis is much more spread out in the case of the signal from the Earth than from the Sun, with many configurations giving values of $\Phi_{\mu}^{\text {Earth }}$ far below (by many orders of magnitude) the experimental sensitivity. The strong attenuation of $\Phi_{\mu}^{\text {Earth }}$ for these configurations is due to the fact that for them the capture-annihilation equilibrium in the Earth is far from being established. As for the signals from the Sun, we further note that for all configurations of Fig. 10 the signal is dominated by coherent contributions, in spite of the scarcity of heavy nuclei in the Sun. This makes the discovery potential for the signal from the Sun essentially equivalent to the one for the signal from the Earth, at this representative point. Spin-dependent effects contribute only for a few per cent, at most. In other representative points, the main contribution to the signal may be due to non-coherent cross sections. However, under these circumstances the overall signal is much smaller than the experimental limit.

In all the examples just discussed (Figs. 6-11), referring to the representative value $\tan \beta=53$, we have found that measurements of $\Phi_{\mu}$ provides a useful tool for investigating interesting regions of the supersymmetric parameter space, when the boson $A$ is light, $M_{A}=O\left(M_{Z}\right)$. Unfortunately, but unavoidably, the very fact that $M_{A}$ is small makes it difficult for these supersymmetric configurations to yield a neutralino relic density in the desired range: $0.1 \leq$ $\Omega_{\chi} h^{2} \leq 0.3$. A quite opposite scenario occurs in different, and equally allowed, sectors of the parameter space, such as those examined in Section 4, where $\Omega_{\chi} h^{2}$ is very large (and where the signals $\mathrm{R}$ and $\Phi_{\mu}$ are much below the present and the foreseeable experimental sensitivities). This is typically a scenario of small $\tan \beta$ or it may occur at large $\tan \beta$ with deviations from $m_{0}$ universality (see, for instance, the case illustrated in Fig. 3). It is remarkable that both scenarios are possible in the case of the supergravity-inspired model adopted in the present paper, although it is constrained by the requirement of radiative EWSB. Of course, much wider regions of the parameter space are allowed to both scenarios in the case of the unconstrained MSSM.

\section{Acknowledgements}

This work was supported, in part, by the Human Capital and Mobility Programme of the European Economic Community under contract No. CHRXCT93-0120 (DG 12 COMA) and by the Research Funds of the Ministero dell'Università e della Ricerca Scientifica e Tecnologica. Also a fellowship of the Istituto Nazionale di Fisica Nucleare is gratefully acknowledged by $\mathrm{N}$. Fornengo and one of the Università di Torino by G. Mignola.

\section{References}

[1] J. Ellis, J.S. Hagelin, D.V. Nanopoulos, K. Olive and M. Srednicki, Nucl. Phys. B 238 (1984) 453.

[2] M. White, D. Scott and J. Silk, Ann. Rev. Astron. Astrophys. 32 (1994) 319; M. White and D. Scott, astro-ph/9601170.

[3] J.R. Primack, D. Seckel and B. Sadoulet, Ann. Rev. Nucl. Part. Sci. 38 (1988) 751; P.F. Smith and J.D. Lewin, Phys. Rep. 187 (1990) 203.

[4] G. Jungman, M. Kamionkowski and K. Griest, Phys. Rep. 267 (1996) 195.

[5] R. Bernabei, Riv. Nuovo Cim. 18 (1995) N5; L. Mosca, invited talk at TAUP 95 (Toledo, September 1995), Nucl. Phys. B: Proc. Suppl., to appear.

[6] V. Berezinsky, A. Bottino, J. Ellis, N. Fornengo, G. Mignola and S. Scopel, preprint CERN-TH 95206, Astropart. Phys., to appear.

[7] G.F. Giudice and E. Roulet, Nucl. Phys. B 316 (1989) 429; G.B. Gelmini, P. Gondolo and E. Roulet, Nucl. Phys. B 351 (1991) 623; M. Kamionkowski, Phys. Rev. D 44 (1991) 3021; A. Bottino, V. de Alfaro, N. Fornengo, G. Mignola and M. Pignone, Phys. Lett. B 265 (1991) 57; F. Halzen, M. Kamionkowski and T. Steltzer, Phys. Rev. D 45 (1992) 4439; V.S. Berezinsky, Nucl. Phys. B: Proc. Suppl. 31 (1993) 413 (Proc. Neutrino 92, ed. A. Morales); M. Mori et al., Phys. Rev. D 48 (1993) 5505; M. Drees, G. Jungman, M. Kamionkowski and M.M. Nojiri, Phys. Rev. D 49 (1994) 636; R. Gandhi, J.L. Lopez, D.V. Nanopoulos, K. Yuan and A. Zichichi, Phys. Rev. D 49 (1994) 3691.

[8] A. Bottino, N. Fomengo, G. Mignola and L. Moscoso, Astropart. Phys. 3 (1995) 65.

[9] L. Resvanis, invited talk at TAUP 95 (Toledo, September 1995) Nucl. Phys. B: Proc. Suppl., to appear.

[10] J. Ellis, S. Kelley and D. Nanopoulos, Phys. Lett. B 260 (1991) 131; C. Giunti, C.W. Kim and U.W. Lee, Mod. Phys. Lett. A 6 (1991) 1745; U. Amaldi, W. de Boer and H. 
Fürstenau, Phys. Lett. B 260 (1991) 447; P. Langacker and M. Luo, Phys. Rev. D 44 (1991) 817.

[11] J. Ellis, G.L. Fogli and E. Lisi, CERN preprint TH/95-202 (1995), and references therein.

[12] H.P. Nilles, Phys. Rep. 110 (1984) 1; H.E. Haber and G.L. Kane, Phys. Rep. 117 (1985) 75; R. Barbieri, Riv. Nuovo Cim. 11 (1988) 1.

[13] L.E. Ibañez and C. Lopez, Nucl. Phys. B 233 (1984) 511; J. Ellis and F. Zwirner, Nucl. Phys. B 338 (1990) 317; J. Ellis and L. Roszkowski, Phys. Lett. B 283 (1992) 252; M. Drees and M.M. Nojiri, Nucl. Phys. B 369 (1992) 54; M. Olechowski and S. Pokorski, Nucl. Phys. B 404 (1993) 590; R.G. Roberts and L. Roszkowski, Phys. Lett. B 309 (1993) 329; R. Arnowitt and P. Nath, Brazil Summer School 3 (1993) 63 (hep-ph/9309277); J.L. Lopez, D.V. Nanopoulos and A. Zichichi, Riv. Nuovo Cim. 17 (1994) 1; W. de Boer, R. Ehret and D.I. Kazakov, Karlsruhe University preprint IEKP-KA/9404(1994); G.L. Kane, C. Kolda, L. Roszkowski and J.D. Wells, Phys. Rev. D 49 (1994) 6173; P. Nath and R. Amowitt, Phys. Rev. Lett. 74 (1995) 4592; X. Wang, J. Lopez and D. Nanopoulos, Phys. Lett. B 348 (1995) 105; R. Rattazzi and U. Sarid, Phys. Rev. D 53 (1996) 1553.

[14] M. Olechowski and S. Pokorski, Phys. Lett. B 344 (1995) 201.

[15] N. Polonsky and A. Pomarol, Phys. Rev. Lett. 73 (1994) 2292; Phys. Rev. D 51 (1995) 6532; D. Matalliotakis and H.P. Nilles, Nucl. Phys. B 435 (1995) 115; A. Pomarol and S. Dimopoulos, CERN preprint CERN-TH/95-114 (1995); H. Murayama, Berkeley preprint LBL36962 (1995).

[16] M.M. Boliev et al.(Baksan Telescope), contribution presented by S.P. Mikheyev at TAUP 95 (Toledo, September 1995), Nucl. Phys. B: Proc. Suppl., to appear; S.P. Mikheyev, private communication.

[17] M. Rowan-Robinson, Les Houches lectures (1993); CERN Colloquium (1995).

[18] W.L. Freedman et al., Nature 371 (1994) 757.

[19] N.R. Tanvir, T. Shanks, H.C. Ferguson and D.R.T. Robinson, Nature 377 (1995) 399.

[20] K. Fisher et al., Princeton preprint IASSNS-AST-95-19 (1995); S. Zaroubi, A. Dekel, Y. Hoffman and T. Kolatt, astro-ph/9603068.

[21] A. Dekel, Ann. Rev. Astron. Astrophys. 32 (1994) 371.

[22] N.Hata, R.J.Scherrer, G.Steigman, D.Thomas and T.P.Walker, Preprint OSU-TA 26/94 (1994), Ap. J. Lett., submitted; C.J. Copi, D.N. Schramm and M.S. Turner, Science 267 (1995) 192; Phys. Rev. Lett. 75 (1995) 3981; S. Sarkar, Oxford University preprint OUTP-95-16-P (1995); B.D. Fields, K. Kainulainen, K.A. Olive and D. Thomas, CERN preprint CERN-TH-96-59 (1996).

[23] C.L. Bennett et al., COBE preprint 96-01 astro-ph/9601067.

[24] M.J. Geller and J.P. Huchra, Ap. J. Suppl. 52 (1983) 61.

[25] C. Kochanek, Ap. J. 419 (1993) 12; E. Bunn and N. Sugiyama, Ap. J. 446 (1995) 49; P. Helbig, Proc. of the 17th Texas Symposium, Ann. NY Acad. Sci. 759 (1995) 600.

[26] A. Klypin, S. Borgani, J. Holtuman and J.R. Primack, Ap. J. $444(1995) 1$.
[27] J.R. Primack, J. Holtzman, A. Klypin and D.O. Caldwell, Nucl. Phys. B: Proc. Suppl. 43 (1995) 133.

[28] J.R.Primack, Proc. of 1994 DPF Summer Study on High Energy Physics: Particle and Nuclear Astrophysics and Cosmology in the Next Millenium (Snowmass 94), to be published.

[29] C.J. Copi and D.N. Schramm, astro-ph/9504026.

[30] L. Rolandi, H. Dijkstra, D. Strickland and G. Wilson, representing the ALEPH, DEL-PHI, L3 AND OPAL collaborations, Joint Seminar on the First Results from LEP 1.5, CERN, December 1995.

[31] J. Ellis, K. Enqvist, D.V. Nanopoulos and F. Zwimer, Mod. Phys. Lett. A 1 (1986) 57; R. Barbieri and G. Giudice, Nucl. Phys. B 306 (1988) 63; M. Olechowski and S. Pokorski, Nucl. Phys. B 404 (1993) 590; B. de Carlos and J.A. Casas, Phys. Lett. R 309 (1993) 320.

[32] V. Barger, M.S. Berger and P. Ohmann, Phys. Rev. D 49 (1994) 4908; G.W. Anderson and D.J. Castaño, Phys. Rev. D 52 (1995) 1693; S. Dimopoulos and G. Giudice, CERN preprint TH/95-188 (1995).

[33] P.H. Chankowski and S. Pokorski, preprint MPI-PhT/95-49 (hep-ph 9505308); D. Garcia and J. Solà, Phys. Lett. B 354 (1995) 335; G.L. Kane, R.G. Stuart and J.D. Wells, Phys. Lett. B 354 (1995) 350.

[34] M. Srednicki, R. Watkins and K. Olive, Nucl. Phys. B 310 (1988) 693; J. Ellis, L. Roszkowski and Z. Lalak, Phys. Lett. B 245 (1990) 545 .

[35] G. Gelmini and P. Gondolo, Nucl. Phys. B 360 (1991) 145.

[36] K. Griest, M. Kamionkowski and M.S. Tumer, Phys. Rev. D 41 (1990) 3565; M. Drees and M.M. Nojiri, Phys. Rev. D 47 (1993) 3776.

[37] A. Bottino, V. de Alfaro, N. Fornengo, G. Mignola and M. Pignone, Astropart. Phys. 2 (1994) 67.

[38] E.I. Gates, G. Gyuk and M.S. Turner, FNAL preprint FERMILAB-PUB-95/090-A (1995).

[39] R.A. Flores, Phys. Lett. B 215 (1988) 73.

[40] A. Gould, Ap. J. 321 (1987) 571; 328 (1988) 919; 368 (1991) 610 .

[41] K. Griest and D. Seckel, Nucl. Phys. B 283 (1987) 681.

[42] M. Drees, G. Jungman, M. Kamionkowski and M.M. Nojiri, Phys. Rev. D 49 (1994) 636.

[43] J. Edsjö and P. Gondolo, preprint PAR/LPTHE/95-12.

[44] T. Sjöstrand, Comput. Phys. Comm. 39 (1986) 347; Comput. Phys. Comm. 43 (1987) 367; CERN-TH 6488/92.

[45] S. Ritz and D. Seckel, Nucl. Phys, B 304 (1988) 877.

[46] G. Jungman and M. Kamionkowski, Phys. Rev. D 51 (1995) 328.

[47] J. Ellis, R.A. Flores and S.S. Masood, Phys. Lett B 294 (1992) 229.

[48] T.K. Gaisser, Cosmic Rays and Particle Physics (Cambridge University Press, 1992).

[49] A. Bottino, V. de Alfaro, N. Fornengo, G. Mignola and M. Pignone, Phys. Lett. B 265 (1991) 57.

[50] L. Bergström and P. Gondolo, Uppsala University preprint UUITP-17-95 (1995). 
[51] M. Mori et al. (Kamiokande Collaboration), Phys. Rev. 48 (1993) 5505

[52] M. Ambrosio et al. (MACRO Collaboration), contribution presented by T. Montaruli at TAUP 95 (Toledo, September 1995), Nucl. Phys. B: Proc. Suppl., to appear.

[53] L.B. Bezrukov et al. (Baikal Underwater Telescope), contribution presented by J. Djilkibaev at 'The Dark Side of the Universe' (Rome, November 1995), Proceedings, to appear.

[54] M. Beck (Heidelberg-Moscow Collaboration), TAUP93 Proceedings, Nucl. Phys. B: Proc. Suppl. 35 (1994) 150.
[55] S.P. Ahlen et al., Phys. Lett. B 195 (1987) 603; D.O Caldwell et al., Phys. Rev. Lett. 61 (1988) 510; A.K. Drukier et al., TAUP91 Proceedings, Nucl. Phys. B: Proc. Suppl. A 28 (1992) 293; I.R. Sagdev, A.K. Drukier, D.J. Welsh, A.A. Klimenko, S.B. Osetrov, A.A. Smolnikov, TAUP93 proceedings, Nucl. Phys. B: Proc. Suppl. 35 (1994) 175; M.L. Sarsa et al., Nucl. Phys. B: Proc. Suppl. 35 (1994) 154; D. Reusser et al., Phys. Lett. B 255 (1991) 143. 